\title{
Gradhiva
}

GRADHIV

Revue d'anthropologie et d'histoire des arts

27 | 2018

Sur le vif. Photographie et anthropologie

\section{Cadrage cannibale. Les photographies de Tristes Tropiques}

Cannibalistic Framing. Photographs in Tristes Tropiques

Vincent Debaene

\section{(2) OpenEdition}

\section{Journals}

Édition électronique

URL : http://journals.openedition.org/gradhiva/3531

DOI : 10.4000/gradhiva.3531

ISSN : 1760-849X

Éditeur

Musée du quai Branly Jacques Chirac

\section{Édition imprimée}

Date de publication : 23 mai 2018

Pagination : 90-117

ISBN : 978-2-35744-110-1

ISSN : 0764-8928

\section{Référence électronique}

Vincent Debaene, "Cadrage cannibale. Les photographies de Tristes Tropiques », Gradhiva [En ligne], 27 | 2018, mis en ligne le 23 mai 2020, consulté le 07 janvier 2021. URL : http://

journals.openedition.org/gradhiva/3531; DOI : https://doi.org/10.4000/gradhiva.3531

(c) musée du quai Branly 


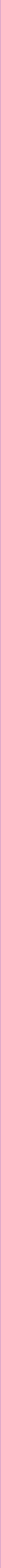




\section{Cadrage cannibale Les photographies de Tristes Tropiques}

par Vincent Debaene

Les premières pages de Tristes Tropiques condamnent avec virulence les récits d'exploration et «albums de photographies » consacrés à des populations prétendument primitives. C'est en particulier l'usage manipulateur du cadrage que Lévi-Strauss dénonce. Pourtant, la publication en 2001 du journal de Luiz de Castro Faria, anthropologue brésilien qui l'accompagnait lors de sa deuxième expédition, a montré que les célèbres photographies des Indiens nambikwara qu'on trouve dans Tristes Tropiques étaient elles-mêmes souvent cadrées de très près, évitant la ligne télégraphique ou le poste missionnaire à l'arrière-plan, sans parler de la présence même des ethnographes en casque colonial. Comment comprendre ce paradoxe, qui frôle la contradiction? Faut-il en conclure, comme beaucoup de critiques l'ont fait, que Lévi-Strauss a été pris à son propre piège, qu'au fond, son livre participe à la mystification qu'il condamne, et que l'esthétique photographique de Tristes Tropiques en est la preuve la plus éclatante? 
1. À partir de la seconde moitié du XIX siècle, de nombreuses disciplines connaissent ces débats qui opposent les vertus du dessin à celles de la photographie et développent, indépendamment les unes des autres, des réflexions sur la possibilité d'une photographie scientifique. Pour le cas de l'anthropologie physique, par exemple, voir Dias 1994.
Les photographies de Tristes Tropiques ne sont «ethnologiques » qu'au sens large: elles ont été de facto reçues comme telles, en raison du contexte où elles sont apparues et de l'identité professionnelle de Claude Lévi-Strauss, mais elles n'ont pas nécessairement été conçues comme telles. Comme il l'a souvent répété lui-même, Lévi-Strauss n'a pas été formé à l'Institut d'ethnologie et n'a pas fréquenté le musée d'Ethnographie du Trocadéro; il n'était pas familier des collections de la photothèque du musée et il était étranger au discours d'exhaustivité et au projet «d'archives de l'humanité» portés par Paul Rivet et Marcel Mauss. II est par ailleurs peu vraisemblable qu'il ait fréquenté les expositions de photographies (Ella Maillart, Pierre Verger, Titaÿna...) que le musée accueillait au début des années 1930 (voir Mauuarin 2015). Autrement dit, les photos de Tristes Tropiques sont sans aucun doute des photos d'ethnologue, prises par un ethnographe sur le terrain, mais il n'est pas certain qu'elles aient été prises avec une visée de documentation ethnologique: dans le livre de 1955, on trouve la photo d'un archer nambikwara (fig. 2), mais quand il s'agit de documenter la position de la main, c'est au dessin que Lévi-Strauss a recours dans ses carnets (fig. 1). Il y a chez lui une méfiance très «classique » à l'endroit de la photographie, qui rappelle les réticences de Franz Boas à l'égard d'un médium dont on soupçonne l'automaticité, l'immédiateté, et qui, envisagé d'abord comme un moyen d'enregistrement, paraît inférieur au dessin en ce qu'il ne distingue pas l'essentiel de l'accessoire et ne mobilise aucune faculté d'intellection ${ }^{\mathbf{1}}$ (voir Joseph 2013).

La formule «cadrage cannibale» ne se trouve pas comme telle dans Tristes Tropiques. Elle fusionne en réalité deux passages du quatrième chapitre, "La quête du pouvoir», distants de quelques pages. On sait que le début du livre condamne avec virulence les récits d'exploration et «albums de photographies" consacrés à des populations prétendument primitives, dont, selon Lévi-Strauss, le public français des années 1950 se repaît avec complaisance. Le quatrième chapitre propose une analyse de cette vogue et dénonce, entre autres, les manipulations photographiques qui dissimulent les détails susceptibles de contredire cette image d'une «humanité vierge" aperçue pour la première fois. Cet usage manipulateur du cadrage est qualifié plus loin de «cannibalisme nostalgique»: pour Lévi-Strauss, ces petits arrangements visuels, en apparence anodins, s'inscrivent dans une dynamique de violence sans merci de l'Occident à l'égard des sociétés amérindiennes.

Pourtant, la publication en 2001 du journal de Luiz de Castro Faria, qui accompagnait Lévi-Strauss lors de sa deuxième expédition, a montré que les célèbres photographies des Indiens nambikwara qu'on trouve dans Tristes Tropiques étaient elles-mêmes souvent cadrées de très près, évitant la ligne télégraphique ou le poste missionnaire à l'arrière-plan, sans parler de la présence même des ethnographes en casque colonial. Comment comprendre ce paradoxe, qui frôle la contradiction? Faut-il en conclure, comme beaucoup de critiques l'ont fait, que Lévi-Strauss a été pris à son propre piège, que son livre, au fond, participe à la mystification qu'il condamne, et que l'esthétique photographique de Tristes Tropiques en est la preuve la plus éclatante?

Nous reviendrons d'abord sur cette dénonciation des «albums de photographies " pour essayer d'en saisir les termes précisément, avant de comparer les photos de Lévi-Strauss et celles de Castro Faria et d'envisager les interprétations possibles d'un tel contraste. Nous tâcherons enfin de faire sens malgré tout de cette apparente contradiction. Mais s'il fallait donner une 


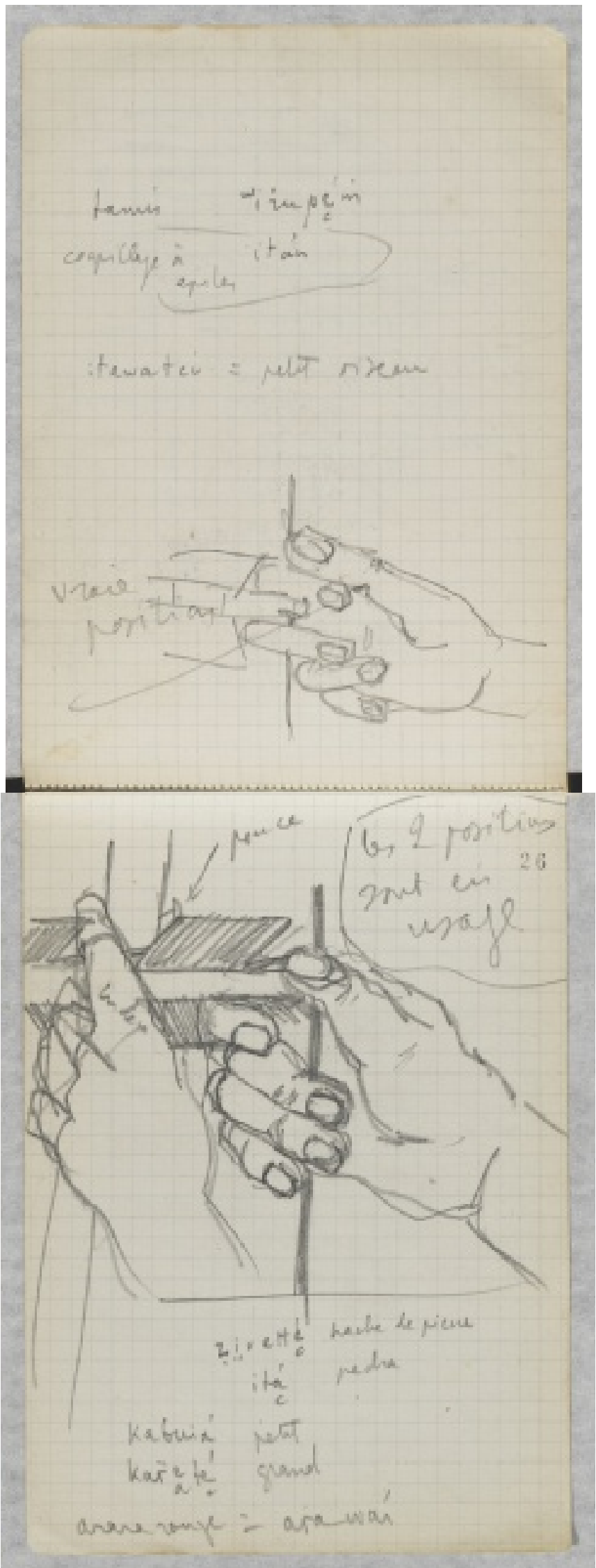

fig. 1

Dessin des mains d'un archer mundé (extrait du carnet «Tupi 1 » et réalisé fin octobre 1938), qui met en évidence la position de la main droite, dite «méditerranéenne ", distincte de celle en usage chez les Bororo et les Nambikkwara. Paris, Bibliothèque nationale de France, NAF 28150(125) f. $25 \mathrm{v}-26 \mathrm{r}$. 
2. Sur la richesse et la variété que recouvre la notion de primitivisme, voir la magistrale introduction de Daniel Fabre au numéro de Gradhiva. Arts de l'enfance, enfance de l'art (Fabre 2009). portée plus générale au propos, ce serait une invitation à la nuance et à davantage de patience ethnographique dans l'étude d'un cas comme celui de Tristes Tropiques. Car l'exposition de la supposée contradiction de Lévi-Strauss relève de deux gestes beaucoup plus larges qui se trouvent en l'occurrence combinés: la dénonciation du primitivisme et ce qu'on pourrait appeler «le jeu du plus malin». La dénonciation du primitivisme est un argument presque aussi ancien que l'ethnologie professionnelle elle-même (pensons aux «lllusions à rebours » de Roger Caillois): il s'agit de condamner l'essentialisme sous-jacent de la démarche ethnologique, d'y déceler une nostalgie des paradis perdus, un mythe des peuples premiers, une «idéalisation de l'autre par inversion systématique des traits négatifs associés à la civilisation occidentale moderne» (L'Estoile 2012: 368). Or le primitivisme est un trop gros concept; il offre une grille de lecture très faible: il est beaucoup plus intéressant d'essayer d'identifier la singularité et l'originalité de la nostalgie de Lévi-Strauss que de montrer en quoi cette nostalgie ressemble à celle de Montaigne, de Rousseau, de Diderot, ou de Sebastião Salgado ${ }^{2}$. Quant au jeu du plus malin, ce fut une sorte de sport dans l'université américaine lorsque la relecture des classiques de l'anthropologie était à l'ordre du jour; au-delà même de l'ethnologie, c'est un réflexe interprétatif profondément ancré dans le rapport aux textes, canoniques ou non, qu'on peut, à l'occasion, retrouver dans certaines réflexions françaises. Le geste n'est pas sans vertu ni efficacité subversive, mais il demeure fondamentalement demi-habile et ne peut en lui-même constituer le terme de l'analyse. Le meilleur exemple de ce petit jeu est sans doute le chapitre que Clifford Geertz consacre à Tristes Tropiques dans Ici et là-bas: l'anthropologue comme auteur, chapitre dont le projet entier consiste à montrer que Lévi-Strauss ne fait pas ce qu'il dit, c'est-à-dire à montrer que la dénonciation des voyages et des récits de voyage est moins une condamnation qu'un déni, car l'ouvrage qui la contient n'est rien d'autre, en fait (nous dit Geertz), qu'un avatar de la «tradition française des récits de voyage ", qui va de Pierre Loti à André Malraux, en passant par André Gide (Geertz 1996: 42). Mais là encore, il semble plus intéressant - non pas plus moral ou plus respectueux, mais simplement théoriquement plus satisfaisant et plus fructueux - de mettre en avant en quoi Lévi-Strauss diffère de Malraux et de Loti. Et un ethnologue aussi expert que Geertz aurait aussi pu se souvenir qu'une fois qu'on a pointé que quelqu'un ne fait pas ce qu'il dit, il reste à s'atteler à la tâche de comprendre ce que réellement il fait.

J'ouvre ces récits d'explorateurs: telle tribu, qu'on me décrit comme sauvage et conservant jusqu'à l'époque actuelle les mœurs de je ne sais quelle humanité primitive caricaturée en quelques légers chapitres, j'ai passé des semaines de ma vie d'étudiant à annoter les ouvrages que, voici cinquante ans, parfois même tout récemment, des hommes de science ont consacrés à son étude, avant que le contact avec les Blancs et les épidémies subséquentes ne l'aient réduite à une poignée de misérables déracinés. Cet autre groupe, dont l'existence, dit-on, a été découverte et l'étude menée en quarante-huit heures par un voyageur adolescent, il a été entrevu (et ce n'est pas négligeable) au cours d'un déplacement hors de son territoire dans un campement 
provisoire, naïvement pris pour un village permanent. Et on a minutieusement gazé les méthodes d'accès, qui auraient révélé le poste missionnaire depuis vingt ans en relations permanentes avec les indigènes, la petite ligne de navigation à moteur qui pénètre au plus profond du pays, mais dont l'œil entraîné infère aussitôt l'existence d'après de menus détails photographiques, le cadrage n'ayant pas toujours réussi à éviter les bidons rouillés où cette humanité vierge fait sa popote. (Lévi-Strauss 2008a: 17)

Tel est l'argument qui fonde, pour Lévi-Strauss, la dénonciation des «albums de photographies» qui occupent les étals des libraires dans la France de l'après-guerre: «par une manipulation qui chez les plus sincères est seulement inconsciente», ces ouvrages «tamisent» la réalité et excluent du cadrage tout ce qui ne confirme pas le «poncif » d'une «humanité vierge» (ibid.). Un mot sur ce très curieux terme de gazer. II s'agit d'un archaïsme, un terme qu'on trouve surtout dans la littérature du $\mathrm{XIX}^{\mathrm{e}}$ siècle et qui signifie "couvrir d'une gaze», «masquer avec de la gaze», et donc «voiler», "dissimuler $^{3}$ ". Lévi-Strauss est probablement l'un des seuls auteurs, sinon le seul, à employer en 1955 le verbe gazer dans cette acception. On peut y voir un signe de plus de son appartenance idéologique et esthétique au XIX $\mathrm{X}^{\mathrm{e}}$ siècle; on peut aussi y reconnaître une analogie qui, sans être jamais explicite, affleure à plusieurs reprises dans Tristes Tropiques et se révèle essentielle pour comprendre le projet même du livre: à savoir le rapprochement entre l'expérience de "gibier de camp de concentration » que Lévi-Strauss connaît en 1941 et celle des Indiens "gibier pris aux pièges de la civilisation mécanique » (Lévi-Strauss 2008a: 10, 29). Nous y reviendrons.

II faut noter également que si, aujourd'hui, on retient que Tristes Tropiques s'ouvre sur une dénonciation des voyages et des récits d'exploration en général, pour un lecteur de l'époque, la cible de Lévi-Strauss est beaucoup plus précise et ne fait aucun doute. L'allusion «aux salles Pleyel cinq ou six fois combles » est claire: ce sont les conférences de l'association Connaissance du monde qui sont visées. Cette association a été créée en 1945; en 1955, elle se targue de réunir «un million d'auditeurs par saison » qui, «non seulement à Paris (où ses premières, à la salle Pleyel, sont devenues l'un des évènements de la vie parisienne), mais dans plus de trois cent cinquante villes de France », assistent aux «conférences d'explorateurs, d'artistes, de chercheurs, de savants [...] accompagnées de la projection de documentaires en couleurs d'une rare qualité ${ }^{4}$ ». Parmi «les explorateurs et les grands voyageurs-cinéastes » que l'association se vante d'avoir fait connaître au public, on retrouve quelques noms connus des ethnologues: Paul-Émile Victor, Georges Condominas, Jean Rouch, Marcel Griaule - auteur en 1946 d'un «Que sais-je? » sur Les Grands Explorateurs, dans lequel il faisait des ethnologues du musée de l'Homme les descendants des «grands explorateurs » d'autrefois, et auquel, vraisemblablement, la célèbre première phrase de Tristes Tropiques fait allusion (voir Debaene 2004).

Vient ensuite le moment de l'interprétation: pour comprendre cette "vogue ", Lévi-Strauss propose de faire un détour par les rites d'initiation des Indiens des Plaines, "car l'ethnographie doit aider à comprendre la mode qui attire vers elle tous ces concours qui la desservent » (Lévi-Strauss 2008a: 27). II propose de tracer un parallèle entre les explorateurs de Connaissance du monde et les adolescents sioux, qui, au moment de la puberté, se lancent
3. Malgré mes recherches, je n'ai pas réussi à savoir si cela correspondait à une technique spécifique de retouche photographique.

4. Texte de présentation du premier des Cahiers Connaissance du monde, $2^{\mathrm{e}}$ trimestre 1955. Dans le numéro du quatrième trimestre 1956, l'association reproduit la diatribe de Lévi-Strauss et lui répond longuement par la voix de Bertrand Flornoy, fondateur et président de la Société des explorateurs français (ou Club des explorateurs) Celui-ci condamne entre autres le dédain du public dont font preuve ces "esprits bien amers et bien naïfs dans leur solitude philosophique - qui tentent d'ironiser sur "le monde vu en Kodachrome"! " (Flornoy 1956 : XXXVII) 


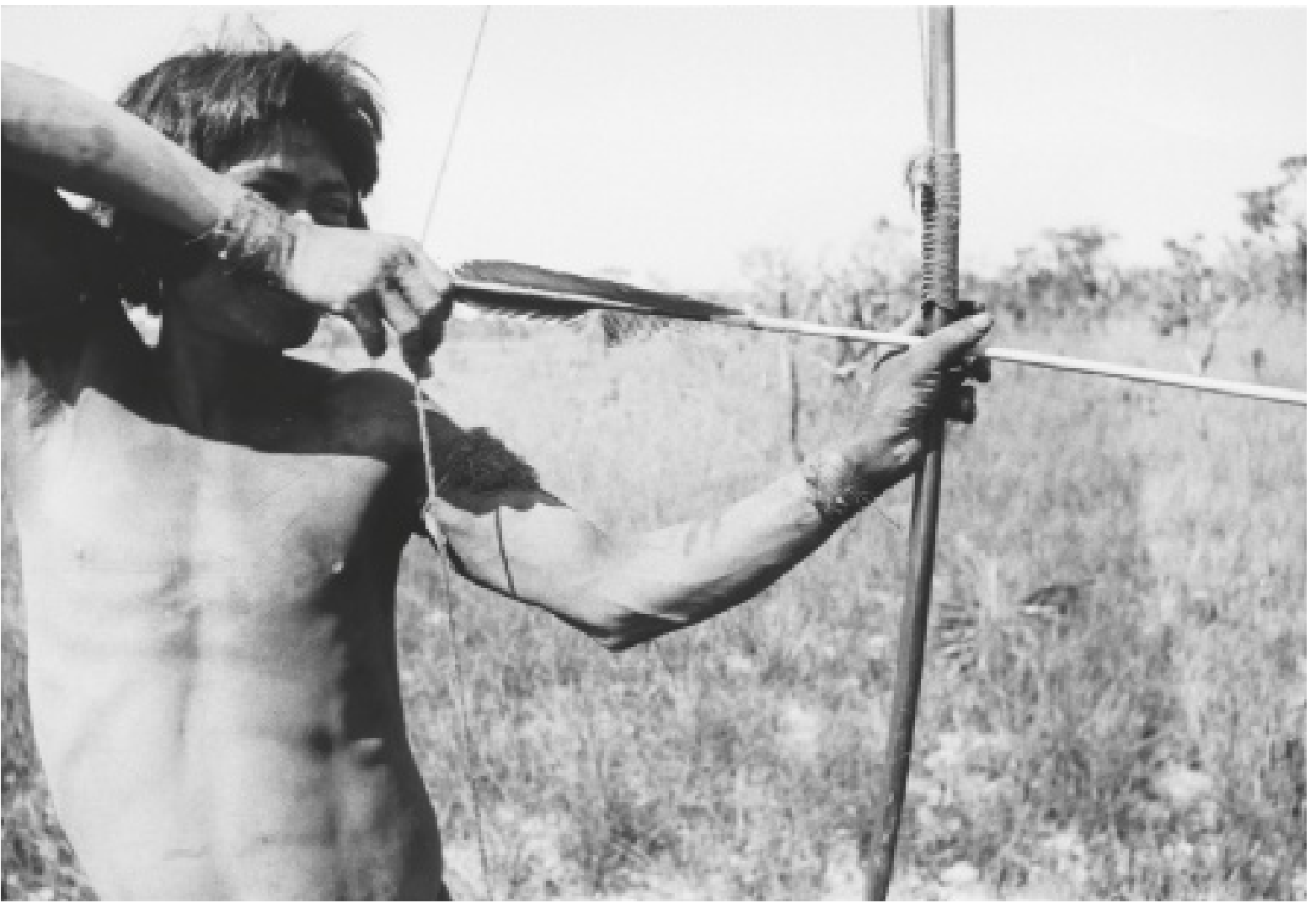

fig. 2

Claude Lévi-Strauss,

Photographie d'un archer

nambikwara, prise très

vraisemblablement à

Utiariti en juin ou juillet

1938 , et qui deviendra

le cliché 28 dans l'édition

originale de Tristes

Tropiques. Paris,

Bibliothèque nationale

de France, NAF

28150(246). 
dans des vision quests et se soumettent à des épreuves physiques et psychologiques à la limite, parfois, du soutenable, dans l'attente d'une illumination qui leur révélera leur esprit gardien, leur pouvoir particulier au sein de la tribu, leur privilège et leur rang. Pour Lévi-Strauss, les conférences de Connaissance du monde relèvent d'un mécanisme semblable, quoique plus naïf, de «quête de pouvoir » et «d'automystification du groupe par lui-même».

C'est le fait de la tentative qui compte et non pas son objet. Comme dans notre exemple indigène, le jeune homme qui, pendant quelques semaines ou quelques mois, s'est isolé du groupe pour s'exposer (tantôt avec conviction et sincérité, tantôt au contraire avec prudence et roublardise, mais les sociétés indigènes connaissent aussi ces nuances) à une situation excessive, revient nanti d'un pouvoir, lequel s'exprime chez nous par les articles de presse, les gros tirages et les conférences à bureau fermé, mais dont le caractère magique est attesté par le processus d'automystification du groupe par lui-même qui explique le phénomène dans tous les cas. Car ces primitifs à qui il suffit de rendre visite pour en revenir sanctifié, ces cimes glacées, ces grottes et ces forêts profondes, temples de hautes et profitables révélations, ce sont, à des titres divers, les ennemis d'une société qui se joue à elle-même la comédie de les anoblir au moment où elle achève de les supprimer, mais qui n'éprouvait pour eux qu'effroi et dégoût quand ils étaient des adversaires véritables. Pauvre gibier pris aux pièges de la civilisation mécanique, sauvages de la forêt amazonienne, tendres et impuissantes victimes, je peux me résigner à comprendre le destin qui vous anéantit, mais non point être dupe de cette sorcellerie plus chétive que la vôtre, qui brandit devant un public avide des albums en Kodachrome remplaçant vos masques détruits. Croit-il par leur intermédiaire réussir à s'approprier vos charmes? Non satisfait encore ni même conscient de vous abolir, il lui faut rassasier fiévreusement de vos ombres le cannibalisme nostalgique d'une histoire à laquelle vous avez déjà succombé. (Lévi-Strauss 2008a: 39-30)

Cet extrait est à la fois typique de son époque et singulier, à deux égards. Il est typique parce qu'il reprend la dénonciation marxisante du spectacle de masse caractéristique des années 1950. L'argument est identique, jusque dans le détail, à celui de Roland Barthes dans les Mythologies, tout particulièrement dans «Bichon chez les Nègres », texte exactement contemporain de la rédaction de Tristes Tropiques puisqu'il paraît d'abord comme «Petite mythologie du mois» dans le numéro de mars 1955 des Lettres nouvelles ${ }^{5}$. «Le courage est un acte formel et creux, écrit Barthes, plus il est immotivé, plus il inspire de respect »; «C'est le fait de la tentative qui compte et non pas son objet ", écrit Lévi-Strauss, en une réflexion qui semble anticiper l'essai final de Barthes «Le mythe aujourd'hui » (qui paraîtra en 1957) et qui montre comment, dans le «mythe», la signification se déplace du littéral vers un signifié idéologique connoté et global, en l'occurrence une négation de I'histoire et de la domination et la réduction de l'Afrique à « un guignol un peu dangereux» (Barthes 1970: 62, voir Debaene 2010: 452-456).
5. L'article de Paris-Match à l'origine du texte de Roland Barthes est lui-même repris d'une conférence Connaissance du monde. Voir Jeannette Fiévet, "Au Nigeria. Chez les Nègres rouges avec Bichon ", Cahiers Connaissance du monde, n³, 1955: 103-114. 
6. Je remercie Frédéric Keck d'avoir attiré mon attention sur cet intertexte.

7. Voir par exemple Les Stars d'Edgar Morin, qui, comme les Mythologies de Barthes, paraît au Seuil en 1957. Morin prétend porter un regard anthropologique sur la production standardisée des stars de Hollywood; il compare les attitudes des fans à celles des "peuples archaïques » à l'égard de leur dieu; il y retrouve " cette magie que nous croyons réservée aux "primitifs" et qui est au cœur même de nos vies civilisées" (Morin 1972: 85 96). Ces fans, décrète-t-il sans s'embarrasser de confirmation empirique, sont "des adolescents" et des femmes des "couches sociales intermédiaires " (ibid: 131-132). De la même façon, le sociologue d'aujourd'hui explique que le «mythe des peuples premiers " perpétué par l'anthropologie flatte "la nostalgie des classes moyennes urbaines " qui en sont à la fois victimes et complices (L'Estoile 2012: 395). Dans tous les cas, il semble inconcevable que des hommes blancs d'âge mûr à capital culturel élevé se laissent abuser par de tels sortilèges.

\section{double page suivante}

fig. 3

Luiz de Castro Faria,

Double page d'Um Outro

Olhar. Diário da expedição

À Serra do Norte, Rio de

Janeiro, Ouro Sobre Azul

Editora Ltda, 2001 (C) Luiz

de Castro Faria / Rio de

Janeiro, MAST - Museu

de Astronomia e Ciências

Afins.
Mais en même temps, le ton de Lévi-Strauss n'est pas celui de la démystification ou de l'ironie, c'est celui de la déploration et d'une prise de conscience absolument tragique à l'échelle des civilisations. On a très tôt comparé Lévi-Strauss à Chateaubriand, mais Chateaubriand regrettait la corruption de l'Indien (“C'est un mendiant à la porte d'un comptoir, ce n'est plus un Sauvage dans ses forêts" [Chateaubriand 1852: 261]). Le constat de Lévi-Strauss est beaucoup plus sombre (dans Tristes Tropiques comme dans d'autres textes des années 1940); il y est question d'anéantissement de formes sociales et culturelles: «abolir », «cannibalisme», «supprimer», «succomber». Rappelons que le livre paraît en 1955: dix ans après la fin de la Seconde Guerre mondiale, et au plus fort de l'inquiétude autour de l'atome, la question de la disparition d'une civilisation par la suppression physique d'une population demeurait d'une actualité angoissante et on aurait tort de réduire cette inquiétude à une grandiloquence mélancolique un peu désuète. Du reste, avant les dernières pages du livre («Le moi n'est pas seulement haïssable, il n'a pas de place entre un nous et un rien "), ce quatrième chapitre est déjà marqué d'un tragique pascalien, la formule "C'est le fait de la tentative qui compte et non pas son objet " évoquant immanquablement le fragment «Divertissement » des Pensées: «lls ne savent pas que ce n'est que la chasse et non pas la prise qu'ils recherchent ${ }^{6}$. " Cette réminiscence annonce déjà la métaphore cynégétique à venir (qui transforme l'explorateur-photographe en chasseur et le sauvage en gibier), de même qu'elle préfigure l'inversion du cannibalisme qui va suivre, puisque dans le fragment "Raison des effets", Pascal avait déjà associé la formule "la chasse plutôt que la prise " à un souvenir des "Cannibales» de Montaigne, essai qui fait de la civilisation occidentale la véritable dévoreuse des sociétés indiennes, elles-mêmes cannibales (Pascal 2004: 120 et 101).

Cette déploration est typique aussi parce qu'elle reprend l'assimilation entre spectacle de masse et primitivité, qu'on trouve fréquemment dans la critique marxiste de la société du spectacle de cette époque ${ }^{7}$. Mais Lévi-Strauss fait un usage complètement inverse du parallèle: la sorcellerie chétive est du côté de Connaissance du monde, non des Indiens des Plaines. C'est la civilisation occidentale qui est accusée de se replier sur des rituels grossiers et sans épaisseur, quand les photographies de Tristes Tropiques semblent illustrer - c'est en tout cas ainsi qu'elles seront reçues et comprises certaines valeurs de délicatesse, de raffinement (pensons aux maquillages caduveo) ou, fameusement, de tendresse, à l'exemple des clichés 35,36 et 37 de l'édition originale («Badinage conjugal», «Tendres ébats...», «... et luttes amicales»): "On devine chez tous une immense gentillesse, une profonde insouciance, une naïve et charmante satisfaction animale, et, rassemblant ces sentiments divers, quelque chose comme l'expression la plus émouvante et la plus véridique de la tendresse humaine." (Lévi-Strauss 2008a: 293)

La publication, en 2001, du journal de Luiz de Castro Faria (fig. 3) - qui, après Dina Lévi-Strauss et Jehan Vellard, est le quatrième membre du "personnel scientifique» de la seconde expédition brésilienne - va modifier cette lecture qui, depuis les années 1950 de pré-décolonisation et de guerre 
froide jusqu'au tiers-mondisme des années 1960-1970, percevait une cohérence entre le discours tragique et les clichés mélancoliques. Il y a d'ailleurs fort à parier que le succès de Tristes Tropiques est en partie attribuable à sa couverture (fig. 4): dès la fin des années 1950 - et contre l'avis de Lévi-Strauss -, elle comporte la photographie du jeune Indien nambikwara, doté d'un labret dans la lèvre supérieure, d'une barrette nasale et dont le regard qu'on suppose aisément mélancolique apparaîtra bientôt comme un emblème des " tristes tropiques 8 ".

Le titre du livre de Castro Faria est explicite: Um outro olhar. Diário da expedição À Serra do Norte. Another Look, selon le titre de la traduction anglaise: il s'agit de proposer un autre regard sur une expédition rendue célèbre par Tristes Tropiques. Très vite, ce qui retient l'attention, c'est le contraste avec les photos de Lévi-Strauss. Certes, la publication, en 1994, de Saudades do Brasil avait déjà créé un effet de perspective saisissant en montrant que la soixantaine de clichés de 1955 faisait partie d'un vaste ensemble ${ }^{9}$. Du coup, se trouvaient mis en valeur le travail de sélection et aussi la continuité temporelle entre les différents épisodes relatés dans Tristes Tropiques. En effet, cinquante-neuf des soixante-trois photographies dans le livre de 1955 représentaient des Indiens; les expéditions en tant que telles étaient à peine documentées (une photo de portage de canoë); les autres épisodes étaient soit non illustrés, soit illustrés de dessins. Les cent quatre-vingts photos de Saudades do Brasil reconstruisent une continuité et intègrent les Indiens dans une séquence narrative: ceux-ci ne paraissent plus isolés sur fond de jungle; on visualise ce que le texte disait déjà, à savoir qu'il a fallu une expédition lourde pour se rendre jusqu'à eux. Et puis on voit l'ethnologue dans deux clichés: ici, prenant un bain avec des enfants nambikwara, là, avec son singe Lucinda dans un campement au bord du rio Machado (Lévi-Strauss 1994: 126, 191). Par ailleurs, le livre «leste» de documents photographiques d'autres moments de Tristes Tropiques, comme les développements sur la croissance urbaine à São Paulo, sur les États brésiliens de l'intérieur, ou sur la «troupe» (camions, bêtes de traits...) qui composait l'expédition. Enfin, effet étrange: on y retrouve des «personnages » qu'on ne connaissait jusqu'alors que par un unique cliché et ceux-ci s'animent, deviennent plus « réels» en quelque sorte.

Mais avec le livre de Castro Faria, quelque chose d'autre se passe: on ne gagne pas simplement en documentation, en épaisseur temporelle ou en variations; on découvre vraiment une autre perspective - um outro olhar sur une expédition quelque peu mythique dans l'histoire de la discipline. Rappelons qu'à l'époque, en 2001, on ne dispose d'aucune des recherches qui, depuis, ont éclairé l'histoire de cette expédition; on peut même dater le début de ces recherches de la publication du journal de Castro Faria, car le texte est précédé d'articles savants dus à des spécialistes brésiliens de l'histoire de l'ethnologie qui situent la fameuse expédition de la serra do Norte dans le contexte de l'époque. Le cœur de l'argumentation de Castro Faria - reprise avec plus ou moins de zèle par les commentateurs - n'est pas dans le texte Um outro olhar, qui est un journal très factuel, sans préface, de l'expédition. On le trouve plutôt dans les entretiens qu'il a donnés, dès les années 1980, mais surtout au moment de la sortie du livre à la fois en France et au Brésil (voir Castro Faria 2001b).
8. C'est la même

photographie qu'on trouve en couverture de la première édition de poche du livre la première, donc, qui soit très largement diffusée et qui paraît en 1962. II s'agit d'un des tout premiers volumes de la collection «Le Monde en 10/18 » qui vient d'être créée au sein de l'Union générale d'éditions, filiale de Plon. L'édition originale de Tristes Tropiques comportait en couverture non une photographie, mais un dessin réalisé par une femme caduveo.

9. Saudades do Brasil est un livre de photographies grand format, qui recueille près de deux cents parmi les trois mille clichés rapportés de ses expéditions brésiliennes par Claude Lévi-Strauss. Ces photos sont brièvement légendées, et l'ensemble est précédé d'une courte préface de Lévi-Strauss. L'initiative du livre revient en partie à Monique Lévi-Strauss, qui tenait absolument à ce que son époux légende les trois mille négatifs avant que la mémoire ne lui fît défaut, ce qui fut une entreprise difficile (quoique de moins en moins, au fur et à mesure que la mémoire lui revenait) qui dura trois mois. À cette occasion, les négatifs furent tous tirés en format $13 \times 18 \mathrm{~cm}$ par Monique Lévi-Strauss, et comme certains d'entre eux

frappaient par leur beauté, il fut décidé d'en faire un livre. Les tirages finaux des cent quatre-vingts clichés sélectionnés sont de la main de Mathieu

Lévi-Strauss, qui avait accumulé une longue pratique du développement et du tirage argentiques depuis son jeune âge (Monique Lévi-Strauss, communication personnelle, octobre 2017). 



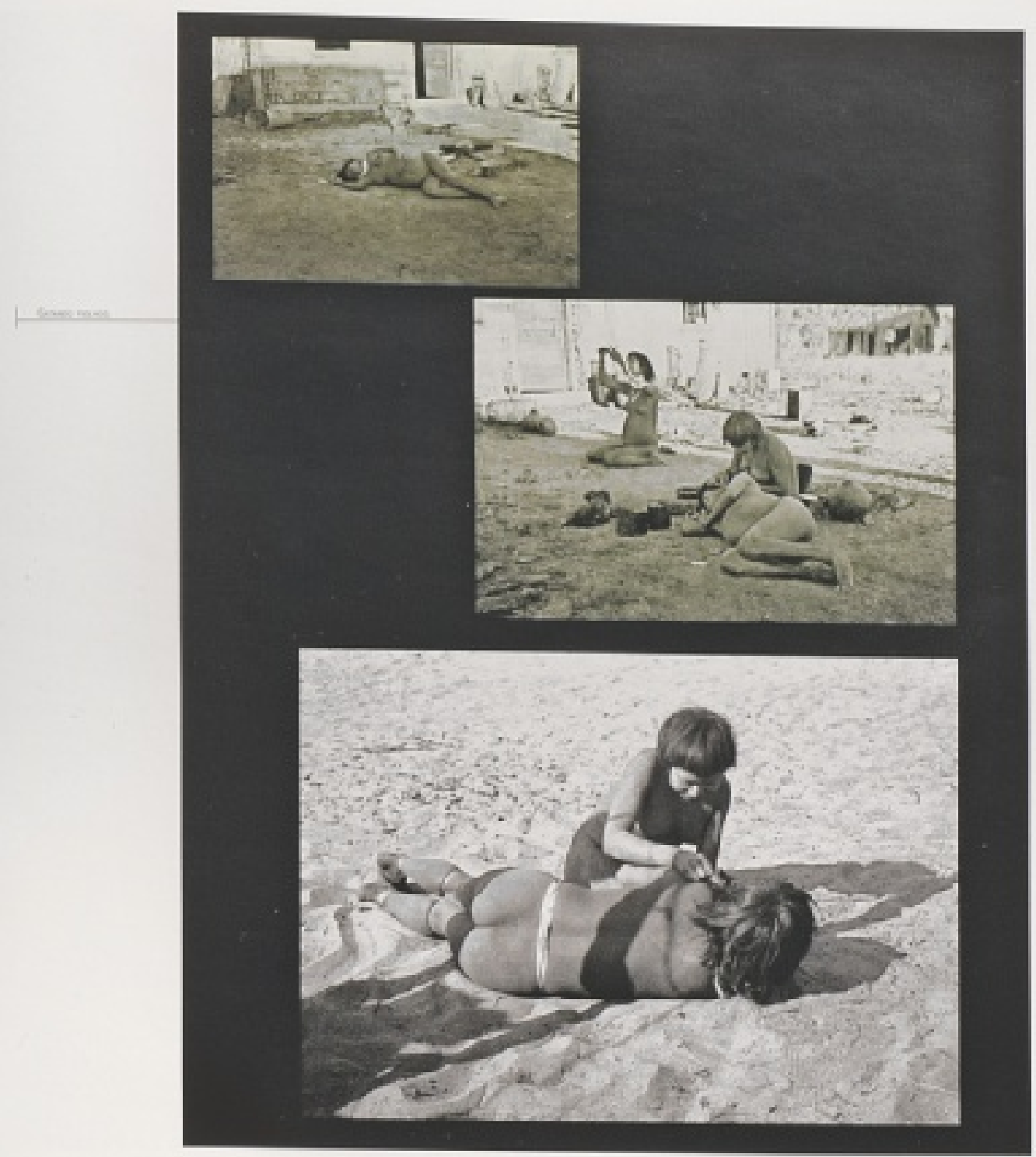




\section{ci-contre}

$\mathrm{fig}=4$

Claude Lévi-Strauss, couvertures de différentes éditions de Tristes Tropiques. Paris, éditions Plon. Avec l'aimable autorisation d'EDI8.
Dans ces entretiens, Castro Faria souligne avec véhémence que Lévi-Strauss n'était pas un homme de terrain, que l'expédition a été un échec "du point de vue ethnographique», et que Lévi-Strauss a gommé les conditions de la rencontre avec les Nambikwara. Castro Faria n'accuse pas explicitement l'anthropologue de tromperie, mais il répète avec insistance que l'expédition demeurait trop peu de temps auprès des groupes nambikwara pour une réelle observation (quelques jours seulement à chaque fois) et que ces derniers ont été pour l'essentiel «rencontrés» et pris en photo non dans leurs villages, mais pendant la période nomade, à proximité de postes télégraphiques de la ligne Rondon. Le texte de Tristes Tropiques est pourtant très explicite sur ce point, mais il est facile, à partir des entretiens de Castro Faria, d'accuser Lévi-Strauss d'avoir voulu faire paraître les Nambikwara comme plus primitifs qu'ils ne l'étaient et de lui appliquer presque littéralement la critique que lui-même adresse aux récits d'explorateurs: lui qui affectait de savoir détecter, avec son œil entraîné, les petites manipulations des voyageurs de Connaissance du monde n'aurait finalement rien fait d'autre, omettant judicieusement à son tour certains détails révélant que le groupe "a été entrevu (et ce n'est pas négligeable) au cours d'un déplacement hors de son territoire dans un campement provisoire, naïvement pris pour un village permanent ».

II semble que les propos de Castro Faria (qu'il avait déjà tenus dans un entretien à Libération en 1988) aient suscité un léger malaise dans la profession à la parution du livre, en raison sans doute d'une aigreur et d'une virulence qui finissent par paraître suspectes. Cela se traduit entre autres par - fait remarquable - non pas une, mais deux recensions dans la revue L'Homme. On doit la plus importante à Michel Perrin, qui met face à face certains clichés de Saudades do Brasil et d'autres d'Um outro olhar. Le contraste est frappant: la «femme enceinte assoupie» (cliché 40 de l'édition originale de Tristes Tropiques repris dans le livre de 1994) reparaît dans Um outro olhar, au centre d'un plan large cette fois, qui révèle la proximité d'une maison dont on aperçoit la porte et le mur en torchis (fig. 3). II est vrai que dans le texte de 1955, le fait que les Nambikwara «dorment par terre et nus » est associé à leur ignorance du hamac et à «l'indigence de [leur] culture matérielle", qui est particulièrement frappante en période sédentaire; on peut donc comprendre la surprise suscitée par un tel arrière-plan (Lévi-Strauss 2008a: 275). Le "sport d'équipe joué avec un ballon en caoutchouc " auquel les Nambikwara se livrent à l'occasion n'était que très fugitivement évoqué dans Tristes Tropiques, mais il était représenté dans Saudades do Brasil par une scène saisie au vol, «sur un terrain dégagé qui donne bien l'idée de l'aspect désertique propre à certaines parties du territoire" (Lévi-Strauss 1994: 127). Des photographies d'Um outro olhar prises de toute évidence au même moment révèlent là encore la proximité d'un poste télégraphique et de poteaux à l'arrière-plan. Plus généralement, les clichés de Castro Faria sont pris de plus loin et donnent une idée du décor qui peut parfois contredire l'image des «bandes err[ant] à travers la savane", semblables "à une colonne de fourmis [...] marchant en file à travers les hautes herbes, chaque femme encombrée par sa hotte en vannerie claire, comme les fourmis le sont parfois de leurs œufs" (Lévi-Strauss 2008a: 271, 274). Enfin, détail capital, sur les clichés de Castro Faria, on voit Lévi-Strauss: chemisette blanche et shorts foncés, casque "colonial», foulard autour du cou, lunettes et barbe - échangeant avec des Nambikwara, eux-mêmes 

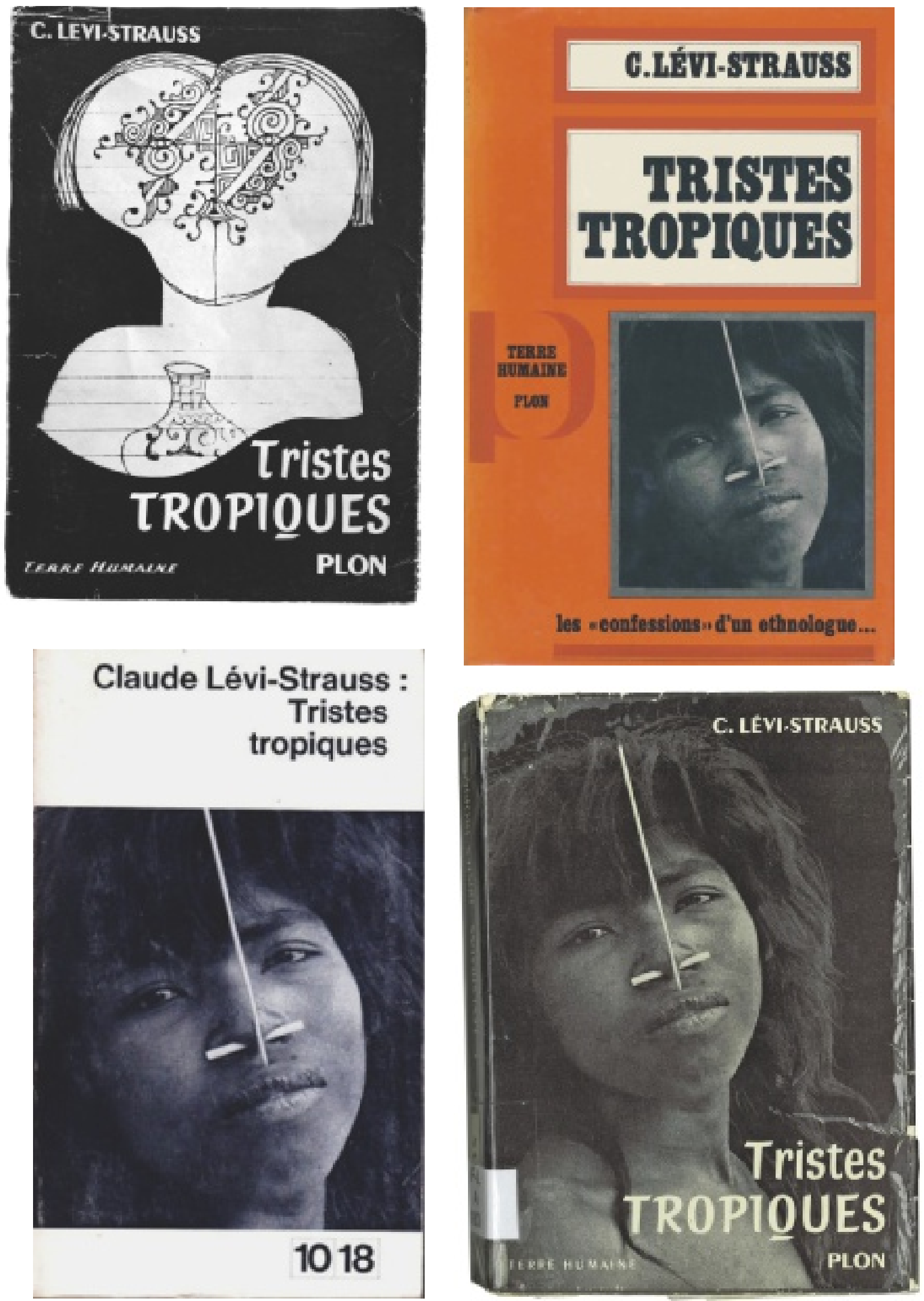
10. La plupart des pages d'Um outro olhar ne comportent pas de folio. presque nus, hormis quelques ornements sur le visage, autour du cou, des biceps et de la taille, à quoi s'ajoute «un pompon de paille accroché quelquefois à la ceinture au-dessus des parties sexuelles " (Lévi-Strauss 2008a: 303) [.fig. 5]. Non seulement on voit Lévi-Strauss, mais on peut même l'observer prendre des photos, et on comprend tout particulièrement que, à défaut de zoom, certains des célèbres plans serrés de Tristes Tropiques supposaient une proximité physique entre le photographe et son sujet qui n'a pas grand-chose à voir avec l'échange équilibré qui préside en théorie au portrait, fondé sur le consentement informé du sujet photographié. Ainsi peut-on assister à la prise du cliché de l'archer nambikwara, pour lequel Lévi-Strauss, Leica vissé à l'œil et visiblement concentré sur la mise au point, se tient à quelques centimètres de l'Indien bandant son arc et à qui il a sans doute demandé de prendre la pose (Castro Faria 2001a ${ }^{10}$ ).

Dans sa recension d'Um outro olhar pour L'Homme, Michel Perrin ne cède pas à la dénonciation et tente de maintenir un équilibre dans la comparaison: assistant au Musée national de Rio de Janeiro, Luiz de Castro Faria est tourné vers l'archéologie, explique-t-il. II a donc privilégié les techniques, la culture matérielle et le milieu naturel; il a préféré «les plans larges, sans les recadrer semble-t-il lors du développement », «laissant souvent apparaître au fond un décor ingrat » : poteaux télégraphiques, bâtiment de mission, clôture pour le bétail. II s'agit de «documents bruts en quelque sorte» (Perrin 2003: 294). Lévi-Strauss, «au contraire, a privilégié l'humain, il l'a isolé »: le «cadrage en général serré, plus "moderne", donne une grande force à ses images et gagne aussi en exotisme »; les photos de Lévi-Strauss ont une "valeur esthétique » et proposent un «émouvant témoignage sur le passé proche d'humanités condamnées et sur les beautés particulières qu'elles ont su sécréter» (ibid).

Pour d'autres, en revanche, la déception est à la mesure de l'enchantement initial (et c'est à ce genre de témoignages qu'on saisit l'impact imaginaire de Tristes Tropiques sur certains de ses lecteurs): André-Marcel d'Ans lit Um outro olhar comme «Le Tour du monde en 80 jours raconté par Passepartout»; il y voit une salubre opération de «redressement de la vérité ». II analyse les «flagrant[s] exemple[s] de discordance entre la désolante médiocrité de cette expédition et l'image mirifique que Lévi-Strauss en donne dans Tristes Tropiques ". À propos des photographies de Tristes Tropiques, il écrit:

[...] on n'y voit que des visages et des corps indigènes, souvent très beaux, saisis en plans très rapprochés ne laissant entrevoir, à l'occasion, que de rares objets, toujours ethnographiques. Sur les vues de Faria, en revanche, si on reconnaît sans peine les mêmes Indiens, parfois saisis avec une fraction de seconde de décalage par rapport à ceux de Lévi-Strauss, apparaissent régulièrement, en raison de cadrages souvent plus larges et d'un traitement différent de la profondeur de champ, des éléments de décor absents sur les images de Lévi-Strauss. C'est ainsi que, montrant des constructions notoirement non indigènes, des clôtures, des chiens, des chevaux, de la volaille, des boîtes de conserve et autres objets de métal, ainsi que des alignements de poteaux électriques, les photos des Nambikwara prises par Faria révèlent que ces Indiens ont été rencontrés non pas en brousse, mais vautrés sur le sol, dans la cour d'une mission et de stations télégraphiques [...]. (d'Ans 2003: 128) 
Au terme de la lecture, note d'Ans, Lévi-Strauss «n'apparaît plus, sur les photos comme dans les notes de Faria, que comme une sorte de Tintin binoclard (casque colonial, bottes de cuir et revolver à la ceinture), passablement empoté, et régulièrement dépassé par le cours des événements": «Tous ceux que ce livre inoubliable a un jour éblouis s'en sentent profondément floués, amputés d'un quartier de rêve. » (ibid.: 128)

Avec une hauteur de vue un peu affectée, certains ont voulu voir là le signe d'une contradiction indépassable qui est le lot de la discipline, et d'une “espèce de schizophrénie de l'anthropologie » (L'Estoile 2012: 399): nous sommes tous des primitivistes, Lévi-Strauss comme les autres; il nous faut nous accommoder de nos contradictions.

Ces commentaires appellent plusieurs remarques. II faut d'abord distinguer la question du primitivisme supposé de Lévi-Strauss de la question du making of, c'est-à-dire du dévoilement du processus de création et de composition qui a abouti au livre Tristes Tropiques. Dans la déception d'André-Marcel d'Ans, ce qui est en cause est moins le cadrage que la révélation du «dessous des cartes": on prend soudain conscience du contexte; on voit les photos être prises, et surtout, on voit le corps de l'ethnographe, qui nous confronte brutalement à la distance qui le séparait d'Indiens que le texte et les photographies nous avaient rendus si proches. Mais une telle lecture du contraste entre les photographies de Castro Faria et celles de Lévi-Strauss décrit moins une tromperie qu'elle ne révèle une structure de croyance et les dynamiques désirantes suscitées par Tristes Tropiques. On peut penser aux analyses d'Octave Mannoni dans son célèbre article "Je sais bien... mais quand même": il y a des formes de démystification qui sont insupportables. Comme Mannoni l'expliquait à propos de l'initiation de Don Talayesva (Sun Chief), on veut bien "se faire à l'idée" que les katchinas ne sont pas réellement présents dans les cérémonies et que leur personnage est joué par des hommes déguisés, mais pas à n'importe quelle condition; un tel savoir doit s'intégrer à un parcours initiatique qui le rend acceptable (Mannoni 1964). De même, on veut bien être rappelé à un ordre de réalité qui veut que les photos de Tristes Tropiques ont été prises par un homme qui, sur le terrain, avait un corps; on est prêt à admettre que les Nambikwara ont été rencontrés par le biais d'intermédiaires (les missionnaires, les fonctionnaires en poste sur la ligne télégraphique) et au terme d'une expédition lourde et nombreuse (des hommes, des camions, des bœufs), «... mais quand même»: cette brutale révélation visuelle est intolérable. La vraie question porte donc plutôt sur les effets de composition de Tristes Tropiques: comment l'ouvrage a-t-il pu à ce point faire oublier les conditions de sa production? Comment ces photographies ont-elles pu faire oublier à ce point qu'elles étaient prises? II y a là une conséquence du dispositif formel d'ensemble constitué par Tristes Tropiques, et de ce qu'on ne peut désigner autrement que sa nature d'œuvre d'art: l'ouvrage construit une totalité autonome qui, pour le lecteur, constitue une expérience suffisante en elle-même et ne réclame pas d'être référée à une autre réalité; la satisfaction esthétique naît précisément de ce sentiment de totalité et des rapports internes perçus entre les différents 
11. Dans une lettre d'octobre 1960 à Claude Gallimard, Alfred Métraux cite en modèle la qualité de l'illustration et de la photographie de "Terre humaine ", qu'il oppose aux « reproductions photographiques médiocres " et aux "couvertures hideuses" de la collection "L'Espèce humaine", dans laquelle il a publié L'île de Pâques en 1941 et Le Vaudou haïtien en 1958 (voir Debaene 2017). niveaux qui la constituent, au détriment de ceux qu'elle entretient avec le reste: c'est ainsi que le livre fait oublier le processus dont il est le résultat. À l'analyse des effets de l'ouvrage lui-même - intégration en une totalité d'un discours et d'une forme textuelle et visuelle singulière -, il faudrait ajouter une enquête historique sur les conditions de sa réception par des individus particuliers, à un moment singulier de leur parcours, dans un univers intellectuel et idéologique spécifique, car l'amertume de ces lectures est à proportion d'un enthousiasme initial que nombre de jeunes hommes et femmes ont connu: combien d'ethnologues formés dans les années 1960 et 1970 attribuent-ils la découverte de leur vocation à la lecture de Tristes Tropiques? (voir Loyer 2015: 439)

On peut, si on le souhaite, prendre la défense de Lévi-Strauss en montrant d'abord que, non, les boîtes de conserve et les bidons d'essence n'ont pas été évités dans les photographies; ils font même l'objet de commentaires dans Tristes Tropiques et plus tard dans les légendes de Saudades do Brasil (voir par exemple le cliché 19 de l'édition originale de Tristes Tropiques, "La bande nambikwara en voyage... », repris avec d'autres dans Saudades do Brasil [Lévi-Strauss 1994: 156-159]; voir également le cliché 27 «Préparation du curare», repris également en 1994 [ibid. : 124-15]). On peut aussi essayer de montrer que les portraits de jeunes femmes rêveuses ou mélancoliques sont fidèles à une certaine réalité, et en particulier à un «ethos» nambikwara fait de méditation et de daydreaming, qui se manifeste dans les postures corporelles (voir Fiorini 2008). Mais contre les généralités bien-pensantes de la critique de la «nostalgie» de Lévi-Strauss, il importe surtout de considérer les photos de Tristes Tropiques non pas «dans l'absolu » - ce qui n'a pas de sens -, ni telles qu'on les trouve déjà (pour une grande partie d'entre elles) dans La Vie familiale et sociale des Indiens nambikwara (1948), ni telles qu'elles ont été reproduites en cartes postales et dans différentes expositions, mais dans leur contexte, là où elles apparaissent, c'est-à-dire précisément dans un livre paru en 1955, intitulé Tristes Tropiques, écrit dans une conjoncture - personnelle et historique - singulière, par un anthropologue particulier.

Ce contexte, c'est donc au moins deux choses: le paysage éditorial des années 1950 et le livre Tristes Tropiques. On sait peu de choses des échanges entre Lévi-Strauss et Jean Malaurie, le directeur de la toute nouvelle collection "Terre humaine», qui fera bientôt de la photographie de qualité - et tout particulièrement de la couverture ornée d'un portrait noir et blanc l'un des «marqueurs» de sa collection ${ }^{\mathbf{1 1}}$. Les correspondances conservées par Lévi-Strauss montrent que celui-ci ne faisait pas de l'illustration une priorité, "convaincu que l'intérêt (s'il y en a un) de [son] livre sera[it] plus "littéraire" que "documentaire" ": «En acceptant des in-textes abondants, je ne ferai donc que satisfaire à votre désir: à vous d'en fixer le nombre», écrit-il à Malaurie en janvier 1955. «Pour [les clichés hors-texte] aussi, il faudra que vous me donniez un chiffre car en ce qui me concerne, une dizaine suffirait. » (Voir Debaene 2008: 1697) Faute de connaître plus précisément les discussions entre Lévi-Strauss et Malaurie, on peut néanmoins observer les photos elles-mêmes et faire jouer différents contrastes.

Notons d'abord que ces photographies sont en noir et blanc; c'est une évidence (et un trait général d'époque), mais c'est un élément essentiel de la critique de Connaissance du monde dont, rappelons-le, la «sorcellerie chétive» «brandit devant un public avide des albums en Kodachrome» (nous soulignons). De fait, la qualité des images de couleur joue un rôle central 


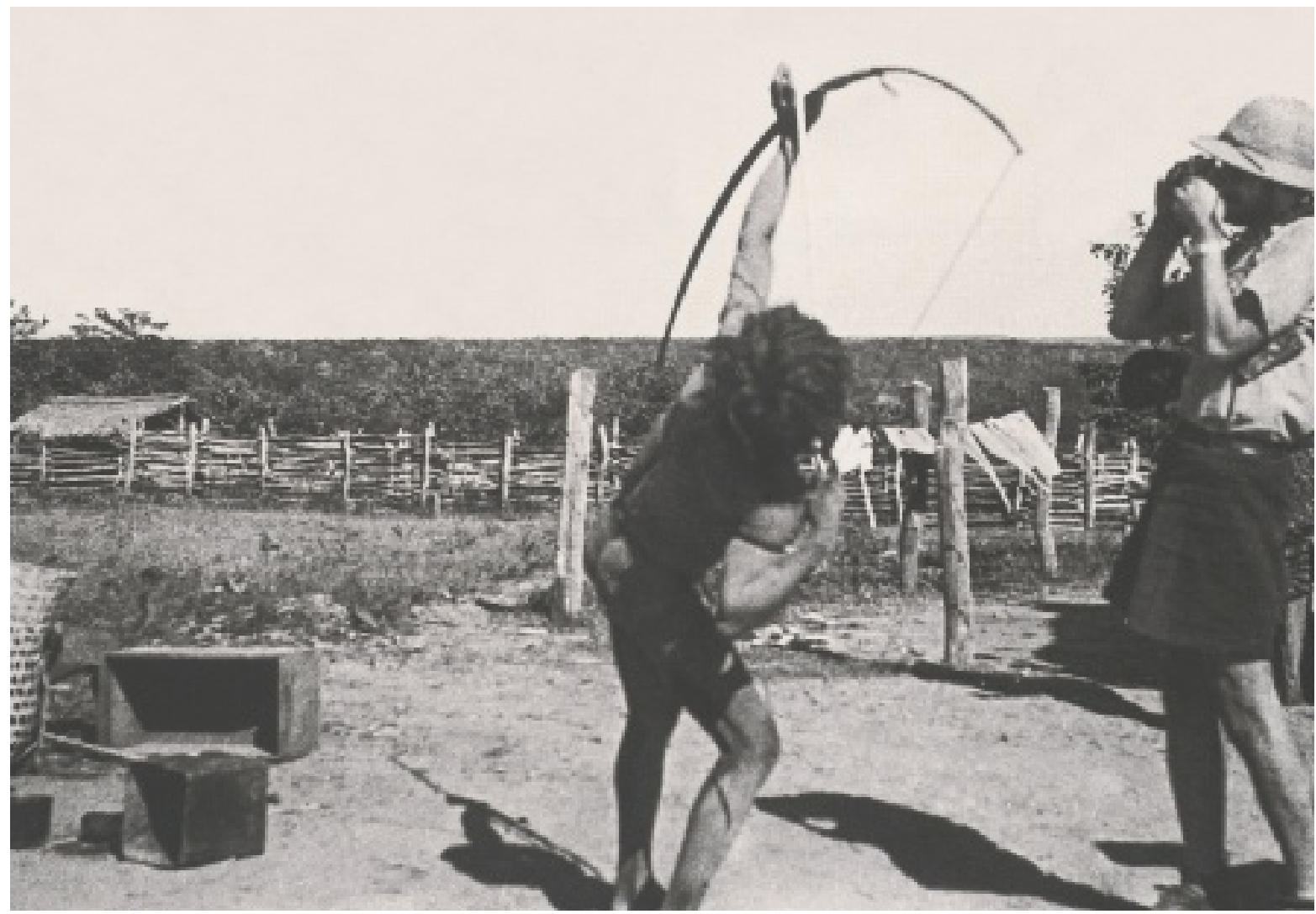

fig. 5

Luiz de Castro Faria,

Claude Lévi-Strauss

photographiant des Indiens

nambikwara, 22 octobre

1938 @ Luiz de Castro

Faria / Rio de Janeiro,

MAST - Museu de

Astronomia e Ciências

Afins. 

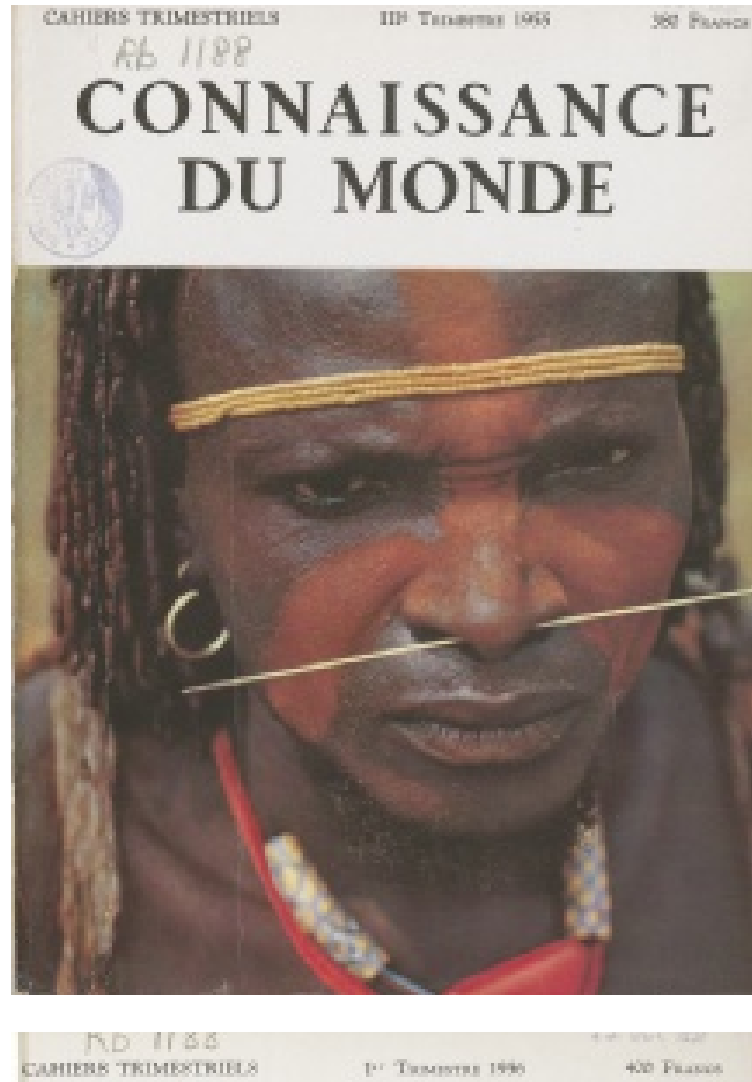

\section{CONNAISSANCE DU MONDE}

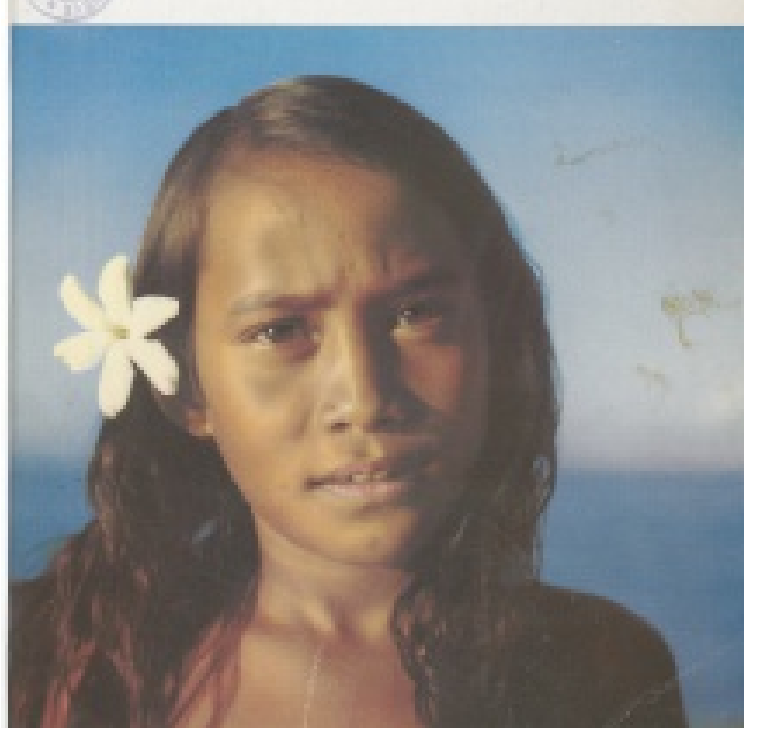

CAranens tamestrates

III" Thasures ips

400 Nusa

Pb 1188

\section{CONNAISSANCE DU MONDE}

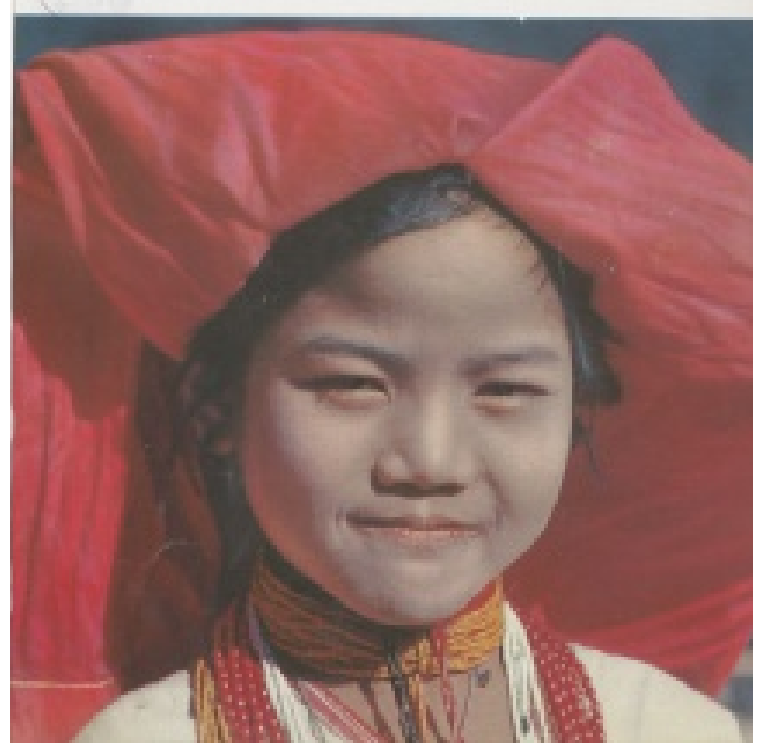

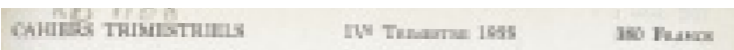

\section{CONNAISSANCE DU MONDE}

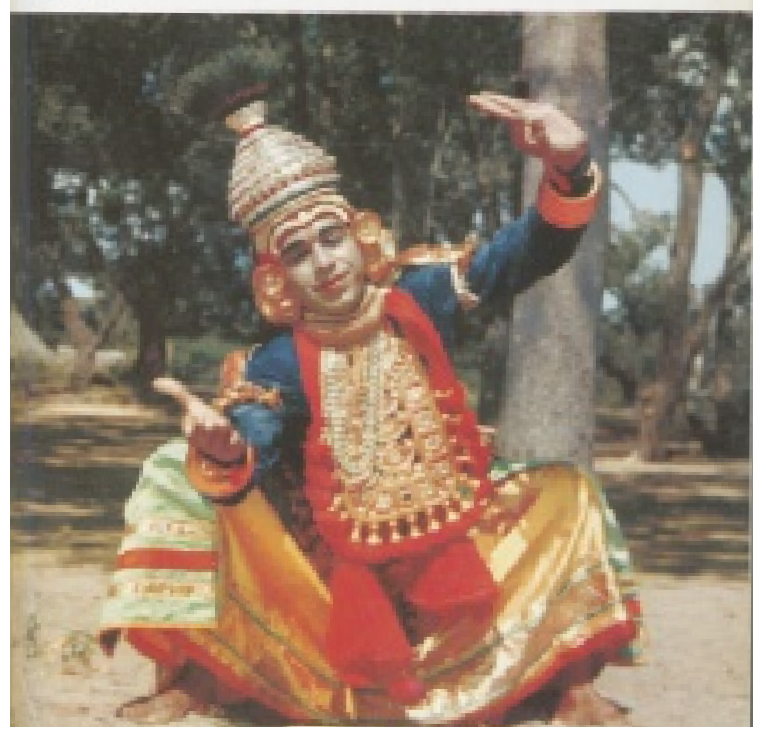


dans le discours publicitaire de l'association, et Lévi-Strauss a raison de l'attribuer à la diffusion du procédé Kodachrome, procédé "soustractif» et non «additif», à la différence de son prédécesseur, l'autochrome, dont I'usage était trop complexe pour être étendu à un large public ${ }^{12}$. Or l'essor de cette technique rencontre de nombreuses résistances dans les années d'après-guerre; elles sont résumées par Barthes dans la mythologie «Continent perdu» (parue initialement en mars 1956): “Le procédé d'irresponsabilité est clair: colorier le monde, c'est toujours un moyen de le nier (et peut-être faudrait-il ici commencer un procès de la couleur au cinéma). " (Barthes 1970: 153) La dénonciation s'inscrit dans une tradition aussi vieille que les premiers essais de photographie en couleurs (qui datent du milieu du $\mathrm{XIX}$ siècle), et elle tient à plusieurs facteurs: au fait que le coloriage est la forme la plus ancienne de retouche photographique; au fait que les photos en couleurs ont d'abord circulé dans des espaces populaires et non savants, et que leur est associée une image de divertissement incompatible avec le sérieux de la science; à un contexte de guerre froide, enfin, et à une association entre couleur et production hollywoodienne: la couleur ne peut ni faire l'objet d'un travail artistique ni avoir de valeur documentaire, elle est l'indication d'une production vulgaire et «purement commerciale» (voir Boulouch 2005; 2011: 85-124; Le Forestier 2013). La couleur est très généralement associée à la dramatisation et au chromo; c'est un procédé «rhétorique» en quelque sorte, qui attire l'attention sur l'image mais distrait du sujet.

Par ailleurs, les photos de Tristes Tropiques ne sont pas retouchées, comme c'est l'usage non seulement dans les Cahiers Connaissance du monde et autres publications populaires, mais aussi dans la plupart des ouvrages ethnographiques de l'époque (la collection «L'Espèce humaine » de Gallimard, par exemple) où le soulignement des contours et l'accentuation des contrastes sont souvent très apparents, même pour un œil non entraîné. Elles privilégient très majoritairement un angle de prise de vue horizontal (ou en légère plongée dans le cas des Nambikwara, souvent saisis en position accroupie ou couchée), qui, par le face-à-face qu'il suggère, met l'accent sur la psychologie et la qualité de sujets des individus représentés. Ceux-ci sont d'ailleurs parfois nommés, à l'exemple de Taperahi, le chef tupi-kawahib, dont Lévi-Strauss nous donne un saisissant portrait en gros plan, qu'il revient au lecteur d'associer à la longue description du chapitre XxxIII et particulièrement à la scène finale de transe, au cours de laquelle Taperahi se livre à une extraordinaire représentation solitaire dans laquelle il incarne «une douzaine de personnages", chacun "distingué par un ton de voix spécial » (Lévi-Strauss 2008a: 378). Par rapport à l'abondante production photographique des Cahiers Connaissance du monde, les clichés de Lévi-Strauss se signalent en tout cas par un refus du spectaculaire et de toute composition picturale "expressive» (alors que la photographie exotique fait un usage récurrent des effets de mise en scène par étagement des plans et de la contre-plongée qui à la fois grandit et éloigne les sujets).

Comme nous l'avons dit, il n'est pas sûr que la photographie ethnographique de l'entre-deux-guerres constitue une référence pour Lévi-Strauss. La comparaison avec les publications ethnographiques des années 1920 et 1930 fait néanmoins apparaître quelques contrastes intéressants. D'abord, Lévi-Strauss ne figure pas sur les photos, à la différence de Bronislaw Malinowski dans Coral Gardens ou des ethnographes de la mission DakarDjibouti dans les diverses publications scientifiques postérieures à l'expédition.
12. L'autochrome est employé par exemple par les opérateurs des "Archives de la planète" d'Albert Kahn (1909-1931). Quoique plus didactique et beaucoup moins diffusée, cette vaste entreprise placée sous la direction scientifique de Jean Brunhes compte parmi les préfigurations de l'ethnographie spectaculaire de Connaissance du monde. Voir Castro 2008;

Boulouch 2011: 69-73.

\section{ci-contre}

fig. 6

Couvertures de plusieurs Cahiers Connaissance du monde. Bibliothèque de Genève. 
13. En 2005, Lévi-Strauss présente son intérêt passé pour la photographie comme une sorte de hobby adolescent: sur le terrain, dit-il, «je photographiais parce qu'il le fallait, mais avec toujours le sentiment que cela représentait une perte de temps, une perte d'attention. [...] La photographie constitue un métier à part, si je puis dire. Ce que j'ai fait est un travail de photographe au degré zéro. J'ai publié un livre de photos [Saudades do Brasil] parce que, autour de moi, on a beaucoup insisté" (Lévi-Strauss 2005: 21)

14. Je me permets de reprendre ici des analyses que j'ai données ailleurs: voir en particulier la notice de l'édition de Tristes Tropiques en Bibliothèque de la Pléiade (2008: 1701-1710) et L'Adieu au voyage (2010 : 308-345).
En cela, néanmoins, il ne se distingue pas forcément d'autres ethnologues français de l'époque; il ne semble pas qu'il y ait vraiment de règle à cet égard. Ensuite, les sujets sont cadrés de beaucoup plus près que ce n'est l'usage dans la littérature savante contemporaine (pensons aux photos des travaux collectifs dans Coral Gardens). Enfin, ils sont saisis en activité (et non en pose), souvent au cours de moments «non marqués» de la vie quotidienne, alors que s'exprime sur les visages une émotion naturelle, qui ne semble commandée ni par l'occasion (rite ou fête), ni par la solennité du portrait préparé. C'est la combinaison de ces deux facteurs - proximité physique et vie quotidienne - dans une série de variations (tout particulièrement dans la partie «Nambikwara» du cahier photographique final) [fig. 7] qui fait l'originalité des photographies de Lévi-Strauss: elles créent le sentiment paradoxal d'une très grande distance (la nudité, les ornements corporels) et d'une très grande proximité - car le lecteur peut reconnaître quelque chose comme sa propre intimité dans ces sourires, ces rêveries, ces badinages et ces chahuts.

À tout cela, il faut ajouter un dernier élément, à savoir la qualité technique des images. Celle-ci est la conséquence d'une émulation, car la pratique photographique de Lévi-Strauss est prise dans une rivalité joueuse avec le père, Raymond, peintre et portraitiste. (Lévi-Strauss a d'ailleurs souvent dit que la gêne financière de son père était la conséquence de la concurrence du portrait photographique et c'est peut-être l'une des sources de sa méfiance à l'endroit de cette technique.) Quoi qu'il en soit, Raymond Lévi-Strauss prenait des photos comme le faisaient les peintres, non pour le résultat pictural luimême, mais afin d'obtenir des épreuves de contrôle (permettant de vérifier les proportions, les angles, et d'affiner les effets de perspective). Comme Lévi-Strauss l'explique dans l'avant-propos de Saudades do Brasil, le critère de cette rivalité est le piqué de l'image, tout cela sur fond d'émerveillement devant les prouesses techniques du Leica, qui, malgré sa petite taille et sa portabilité, permet d'obtenir des images extrêmement nettes et précises (Lévi-Strauss 1994: 22-23). Cela éclaire deux aspects de la pratique photographique de Lévi-Strauss (qu'il abandonne tout à fait ensuite, après quelques années de passion fugitive, comme on se lasse d'un gadget ${ }^{13}$ ): d'une part, la conviction de son infériorité par rapport aux arts de la représentation; d'autre part, son inscription - mais sur un mode plaisant, non sérieux - dans une tradition picturale noble, celle de la peinture de genre, tout particulièrement du portrait flamand, ce dont témoignent les légendes des photographies: "La fileuse interrompue», «Jeune fille au singe ", «La rêveuse », etc.

Mais il faut enfin et surtout penser ces photos au sein du livre Tristes Tropiques et du processus créateur que fut l'écriture de l'ouvrage pour Lévi-Strauss (sans s'arrêter d'ailleurs à ce qu'il en dit et en pense lui-même). Ce processus joue un rôle absolument central dans l'itinéraire de Lévi-Strauss - et pas seulement psychologique ou confessionnel ${ }^{14}$. Celui-ci s'inscrit dans une trajectoire à la fois existentielle et théorique, qui va faire passer Lévi-Strauss d'un «premier " structuralisme (celui des Structures élémentaires de la parenté) - qui est en fait un «fonctionnalisme structural ", selon les termes de Gildas Salmon (2009: 21) - à un second structuralisme, qui prend pour point de départ la logique des qualités sensibles, logique qui est explorée et exploitée dans Le Totémisme aujourd'hui, La Pensée sauvage et qui est au fondement de toute l'entreprise des Mythologiques. Or, à cet égard, il y a un élément dont on ne peut exagérer l'importance, c'est que Tristes Tropiques 
est écrit quinze ans (et une guerre mondiale) après la deuxième expédition. Lévi-Strauss n'illustre pas de ses clichés le récit d'une expédition entreprise l'année précédente: il insère dans une autobiographie intellectuelle des photographies prises entre quinze et vingt ans auparavant. C'est un geste très différent de celui des explorateurs dont les récits abondamment publicisés paraissent immédiatement au retour en France, très différent aussi de celui d'Alfred Métraux dans Le Vaudou haitien, par exemple, pour qui le dernier séjour sur le terrain précède de peu la parution du livre. En 1954, alors qu'il traverse une période de crise à la fois personnelle, intellectuelle et professionnelle, Lévi-Strauss se lance dans une entreprise qu'on pourrait qualifier de «proustienne (il est donc bien vrai que Tristes Tropiques n'est pas un récit de voyage), qui réorganise le passé en se fondant sur une «quête des correspondances » (Lévi-Strauss 2008a: 111). Ce faisant, il fait l'épreuve en lui-même et sur lui-même de la «logique du sensible», cette même logique que, par la suite, il déploiera dans ses analyses des classifications totémiques et des mythes amérindiens. Et à bien des égards l'écriture de Tristes Tropiques peut se lire comme une expérience du «temps retrouvé», pour reprendre le titre d'un chapitre de La Pensée sauvage. II s'agit, écrit-il à la fin (justement) du chapitre «La quête du pouvoir», de ressaisir la vérité d'une expérience ancienne en faisant confiance au travail de l'oubli qui l'a redessinée au mépris de la succession chronologique des jours et des étapes:

[...] les temps et les lieux se heurtent, se juxtaposent ou s'inversent, comme les sédiments disloqués par les tremblements d'une écorce vieillie. Tel détail, infime et ancien, jaillit comme un pic; tandis que des couches entières de mon passé s'affaissent sans laisser de trace. Des événements sans rapport apparent, provenant de périodes et de régions hétéroclites, glissent les uns sur les autres et soudain s'immobilisent en un semblant de castel dont un architecte plus sage que mon histoire eût médité les plans. (Lévi-Strauss 2008a: 32)

Par la remémoration, une logique immanente à l'expérience passée se fait jour, fondée sur les rapprochements entre des expériences sensibles séparées dans le temps et l'espace. Mais cette conquête d'une intelligibilité accordée au sensible a un prix: elle suppose que le flux historique soit cantonné dans un espace propre: l'architecture que dévoile le récit est plus sage que mon histoire, dit Lévi-Strauss. II faut donc que la succession historique soit sinon reversée du côté de la contingence, en tout cas circonscrite, contenue dans un domaine propre, irréductiblement hétérogène à la nécessité logique. L'écriture de Tristes Tropiques est l'opérateur qui va permettre la séparation de deux espaces. D'un côté, la quête d'intelligibilité à partir des vestiges qui surnagent hors d'une histoire catastrophique (et dans l'esprit de Lévi-Strauss le génocide des Indiens d'Amérique n'est pas essentiellement différent de l'extermination des Juifs d'Europe) - quête dont le principe est donné dans La Pensée sauvage et dont le résultat occupera les quatre tomes des Mythologiques, qui s'appuient sur les mythes et tout ce que la collecte ethnographique a pu recueillir comme témoignages des usages anciens, antérieurs à la Conquête. De l'autre, les réflexions plus épisodiques sur l'entropie et la violence, qui feront l'objet de Race et histoire, Race et culture, mais aussi de certains articles du Regard éloigné ou de certains passages d'Histoire de Lynx. double page suivante

fig. 7

Claude Lévi-Strauss,

Double page de Tristes

Tropiques. Paris, éditions

Plon, 1955. Avec l'aimable autorisation d'EDI8. 


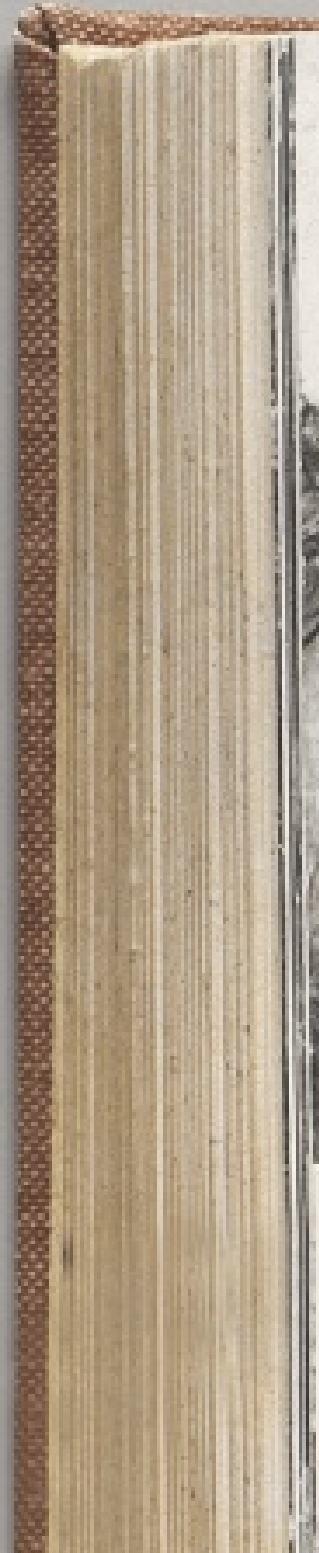

$$
\text { wit }
$$

Fon 2.

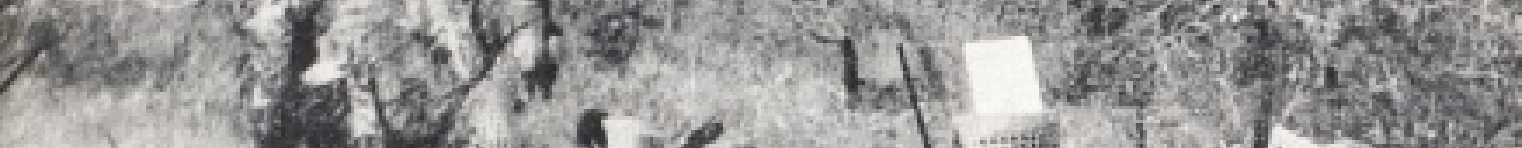

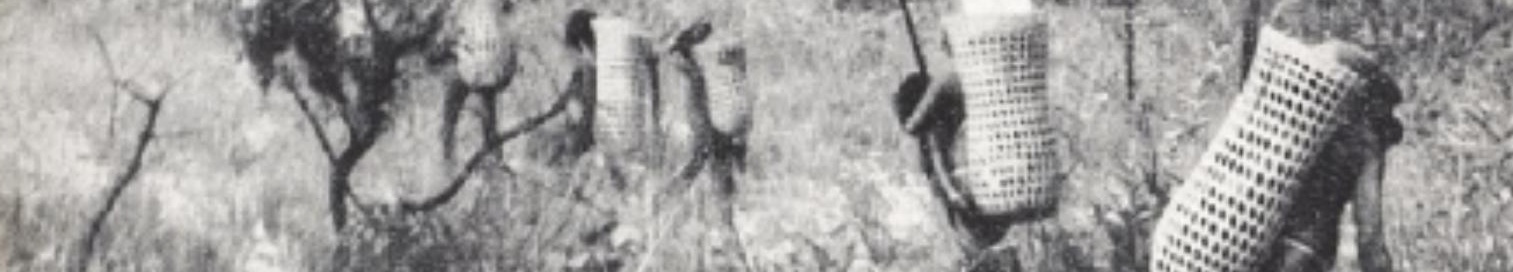
-

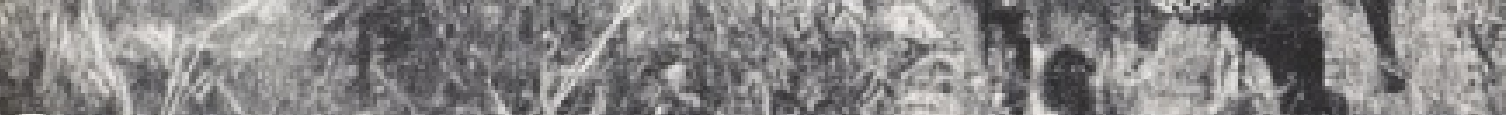

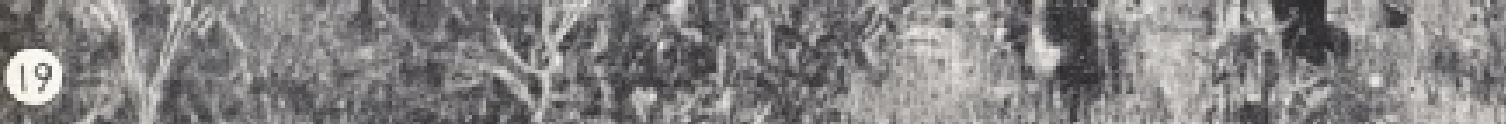

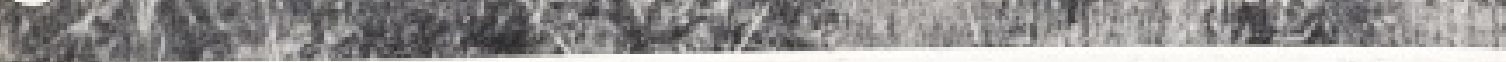

部
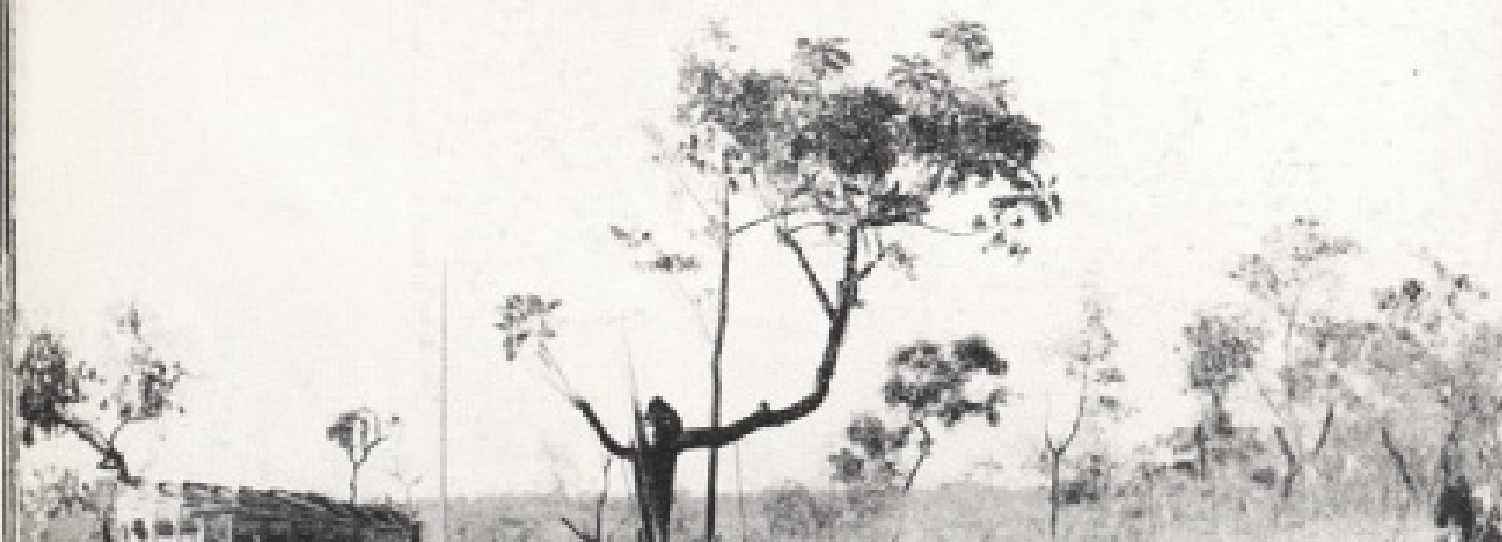

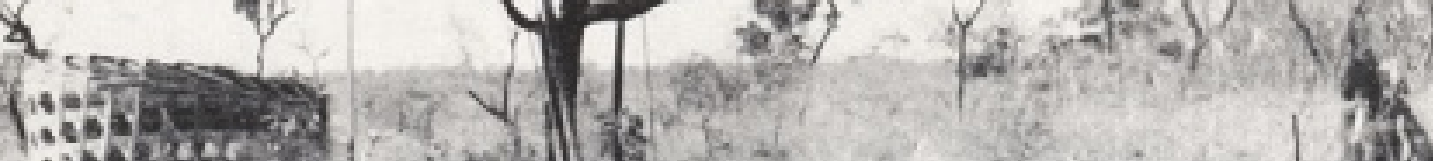

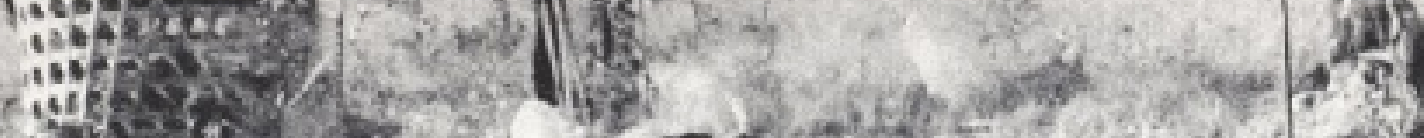
(5)

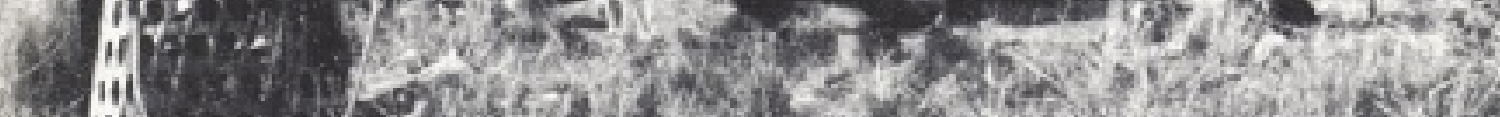

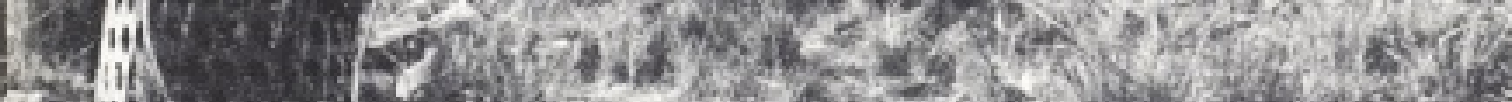
Se. 11 . P. 20\% .

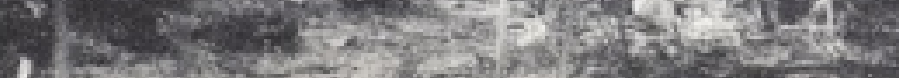

. 


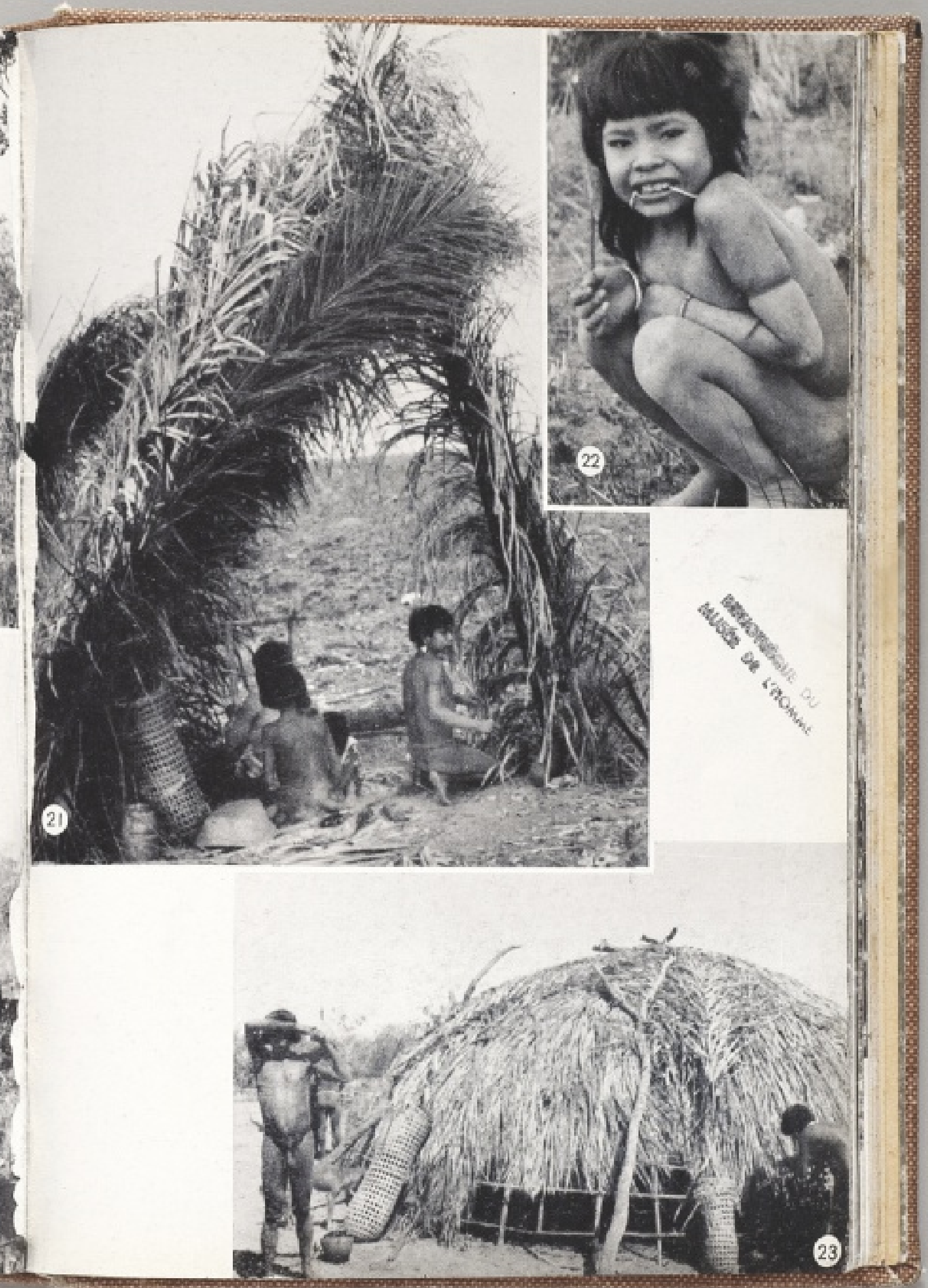


15. II s'agit d'une photo de Pillar Rock, éperon rocheux à la pointe nord-ouest de l'État de Washington, qui figure dans une version makah du mythe du dénicheur d'oiseaux. Le parallèle entre ce paysage et les formations rocheuses de la chapada où les Bororo dénichent les araras (qu'on trouve photographiées dans Le Cru et le Cuit) illustre la possibilité de lire le mythe de la côte nord-ouest comme une transposition du mythe brésilien (Lévi-Strauss 1971: 137-138).

16. Je ne tiens pas compte évidemment des photographies d'objets ou d'œuvres d'art qu'on trouve dans La Pensée sauvage, La Voie des masques, etc. (et qui ne sont pas de Lévi-Strauss). Saudades do Brasil (comme Saudades de São Paulo, qui paraît en 1996 sans être traduit en français) relève donc bien de la veine historique et "entropologique" de l'œuvre; il s'agit fondamentalement de témoigner d'une disparition, d'autant que l'ouvrage paraît au Brésil immédiatement après Histoire de Lynx - éloge funèbre, plus que célébration, publié à l'occasion du cinq centième anniversaire de la Conquête. La parution du livre joue du reste un rôle non négligeable dans le changement d'image que connaît la figure publique de Lévi-Strauss à partir de la fin des années 1980, moins «pape» du structuralisme que vieillard mélancolique et sage, habitué à considérer le destin de l'humanité dans sa globalité.
Les photographies de Tristes Tropiques sont donc véritablement horstexte dans tous les sens du terme: au sens éditorial et technique, mais aussi au sens où elles ne participent pas à l'ordre intelligible reconstruit par le récit. Dans Regarder écouter lire d'abord, puis dans Saudades do Brasil, Lévi-Strauss s'attarde sur l'infériorité congénitale de la photographie réaliste - qui est d'abord infériorité de la vue par rapport à «l'ouïe et l'odorat, ces sens plus proches de l'âme" (Lévi-Strauss 2008a: 358): à la différence de l'odeur de créosote imprégnant ses malles et capable, cinquante ans après, de ressusciter la chose même, les photographies «laissent l'impression d'un vide, d'un manque de ce que l'objectif est foncièrement impuissant à capter " (Lévi-Strauss 2014: 9). C'est pour cette raison, d'ailleurs, que la référence à la peinture de genre dans les légendes («La fileuse interrompue», etc.) n'est pas sérieuse, car dans l'esprit de Lévi-Strauss, la photographie ne propose qu' «un homologue passif de l'objet», alors que la collerette de dentelle peinte par François Clouet constitue au contraire «une véritable expérience sur l'objet», en «compens[ant] la renonciation à des dimensions sensibles [le volume] par l'acquisition de dimensions intelligibles" (Lévi-Strauss 2008b: 583-584). Les photographies demeurent néanmoins - mais comme signes, simples indices, traces d'un passé révolu à jamais inaccessible aux sens. Elles empêchent au fond de céder à une lecture trop «proustienne » du livre: isolées dans un cahier hors-texte, lui-même placé en fin du volume dans l'édition originale, elles disent que certaines résurrections sont impossibles.

II faut sans doute lire l'abandon de la photographie après Tristes Tropiques en parallèle du retrait de Lévi-Strauss de la politique. À partir de Tristes Tropiques, en effet, celui-ci délaisse une réflexion politique qui constituait pourtant un aspect important de sa pensée et qui l'a occupé de 1943 à 1953 environ: des articles comme «Guerre et commerce chez les Indiens d'Amérique du Sud» (1943) ou «La politique étrangère d'une société primitive» (1949) témoignent d'une conception de l'ethnologue comme expert, aidant à la définition des politiques publiques et prêt à contribuer à la reconfiguration des relations internationales dans un monde bouleversé par le second conflit mondial. Or à partir de Tristes Tropiques et de la décolonisation, cette réflexion est abandonnée-et Lévi-Strauss n'inclut pas dans Anthropologie structurale les articles qui en relèvent: l'ordre logique que l'anthropologue va s'attacher désormais à mettre au jour sera retrouvé contre l'histoire, conçue fondamentalement comme le lieu d'un affrontement où le déséquilibre des forces est tel qu'il conduit à l'anéantissement des collectivités qu'elle met en présence, dispersant des formes symboliques dont on ne peut plus recueillir que des fragments.

C'est le même mouvement qu'on observe pour la photographie. À l'exception de quatre clichés en rapport avec le mythe bororo du dénicheur d'oiseaux dans Le Cru et le Cuit, et d'un autre dans L'Homme nu (dont Lévi-Strauss n'est pas l'auteur, mais qui, selon lui, "fait heureusement pendant» à la première planche du Cru et le Cuit ${ }^{15}$ ), on ne trouvera plus de photographies dans l'œuvre postérieure de Lévi-Strauss avant Saudades do Brasil ${ }^{16}$. Lévi-Strauss privilégiera désormais les dessins, et particulièrement les dessins 
anciens, "souvent mieux attentifs que les modernes aux aspects sensibles des choses" (Lévi-Strauss 1983: 292). Ce n'est donc pas le cadrage qui est cannibale, mais l'histoire elle-même. Et la photographie - qui dans son principe même contient une temporalité linéaire et irréversible (entre la «prise » et la révélation, entre le tirage et l'exposition) - ne subsiste dans Tristes Tropiques que comme signe de l'histoire, c'est-à-dire signe de ce qui a été et ne reviendra plus. 


\section{Ans (d'), André-Marcel}

2003 "La tristesse des Tropiques n'est plus ce qu'elle était ", Sociétal 40: $125-128$

\section{Barthes, Roland}

1970 [1957] Mythologies.

Paris, Seuil.

\section{Boulouch, Nathalie}

2005 "Couleur versus noir et blanc ", Études photographiques 16 [en ligne], disponible sur: http://journals.openedition.org/ etudesphotographiques/726 (consulté le 5 mars 2018).

2011 Le Ciel est bleu: une histoire de la photographie couleur.

Paris, Textuel.

\section{Castro, Teresa}

2008 "Les Archives de la Planète et les rythmes de l'Histoire ", 1895. Mille huit cent quatre-vingt-quinze $54: 56-81$

\section{Castro Faria (de), Luiz}

$2001 a$ Um outro olhar: diário da expedição à Serra do Norte. Rio de Janeiro, Ouro Sobre Azul Editora.

$2001 b$ "Ridentes trópicos », Folha de São Paulo, 16 décembre [en ligne], disponible sur: http:// www1.folha.uol.com.br/fsp/mais/ fs1612200105.htm (consulté le 5 mars 2018).

\section{Chateaubriand (de), François-René}

1852 [1827] Voyage en Amérique in CEuvres complètes de Chateaubriand: augmentées d'un essai sur la vie et les ouvrages de l'auteur, t. VI. Paris, P.-H. Krabbe.

\section{Dias, Nélia}

1994 «Photographier et mesurer: les portraits anthropologiques ", Romantisme 84 : 37-49.

\section{Debaene, Vincent}

2004 «Portrait de l'ethnologue en Lazare", in Michel Izard (dir.) Cahiers de l'Herne 82: 99-107.

2008 Notice de Tristes Tropiques, in Claude Lévi-Strauss, CEuvres. Paris, Gallimard: 1675-1722.

2010 L'Adieu au voyage:

l'ethnologie française entre science et littérature. Paris, Gallimard.
2017 "La collection "L'espèce humaine" : rêverie contre-historique", in André Delpuech, Christine Laurière et Carine Peltier-Caroff (dir.), Les Années folles de l'ethnographie: Trocadéro 28-37. Paris, Muséum national d'histoire naturelle: $830-871$.

\section{Fabre, Daniel}

2009 « "C'est de l'art!": le peuple, le primitif, l'enfant ", Gradhiva 9: 5-37.

\section{Fiévet, Jeannette}

1955 «Au Nigéria. Chez les Nègres rouges avec Bichon ", Cahiers Connaissance du monde, $4^{\mathrm{e}}$ trimestre 1955 : 103-114.

\section{Fiorini, Marcelo}

2008 "Lévi-Strauss' Photographs: An Anthropology of the Sensible Body ", Journal de la Société des américanistes 94-2: 55-67.

\section{Flornoy, Bertrand}

1956 "À chacun son point de vue : les explorateurs", Cahiers Connaissance du monde, $4^{\mathrm{e}}$ trimestre 1956 : XXXV-XXXVIII.

\section{Geertz, Clifford}

1996 [1988] /ci et là-bas: l'anthropologue comme auteur, trad. de l'anglais par Daniel Lemoine. Paris, Métailié

\section{Joseph, Camille}

2013 "L'image sans arrière-plan. Franz Boas, la photographie et les Illustrations", in Michel Espagne et Isabelle Kalinowski (dir.) Franz Boas: le travail du regard. Paris, Armand Colin : 155-176.

\section{Le Forestier, Laurent}

2013 "La couleur, naturellement? Débats sur la couleur en France au sortir de la Seconde Guerre mondiale ", 1895. Mille huit cent quatre-vingt-quinze 71 : 253-274.

\section{L'Estoile (de), Benoît}

2012 "Images des paradis perdus: mythe des "peuples premiers", photographie et anthropologie", Vibrant - Virtual Brazilian Anthropology 9-2: 362-405.

\section{Lévi-Strauss, Claude}

1971 L'Homme nu.

Mythologiques IV. Paris, Plon.

1983 Le Regard éloigné.

Paris, Plon.

1994 Saudades do Brasil.

Paris, Plon

2005 Loin du Brésil : entretien avec Véronique Mortaigne. Paris, Chandeigne.

2008a [1955] Tristes Tropiques, in Claude Lévi-Strauss, CEuvres. Paris, Gallimard: 1-445.

2008b [1962] La Pensée sauvage, in Claude Lévi-Strauss, CEuvres. Paris, Gallimard: 553-872.

\section{Loyer, Emmanuelle}

2015 Lévi-Strauss.

Paris, Flammarion.

\section{Mannoni, Octave}

1964 " "Je sais bien... mais quand même" : la croyance ", Les Temps Modernes 212: 1262-1286.

\section{Mauuarin, Anaïs}

2015 "De "beaux documents" pour l'ethnologie. Expositions de photographies au musée d'Ethnographie du Trocadéro (1933-1935) ", Études photographiques 33: 1-19.

\section{Morin, Edgar}

1972 [1957] Les Stars.

Paris, Seuil.

\section{Pascal, Blaise}

2004 [1670] Pensées (Michel Le Guern, éd.). Paris, Gallimard.

\section{Perrin, Michel}

2003 «Regards croisés.

La photographie, entre donnée et emblème", L'Homme. Revue française d'anthropologie 165: 291-300.

\section{Salmon, Gildas}

2009 Logique concrète et transformations dans l'anthropologie structurale de Claude Lévi-Strauss. Thèse de doctorat en philosophie, Paris, université Panthéon-Sorbonne. page $\mathbf{9 0}$ et ci-contre Luiz de Castro Faria, Claude Lévi-Strauss photographiant des Indiens nambikwara, 22 octobre 1938 (détail) (C) Luiz de Castro Faria / Rio de Janeiro, MAST - Museu de Astronomia e Ciências Afins. 


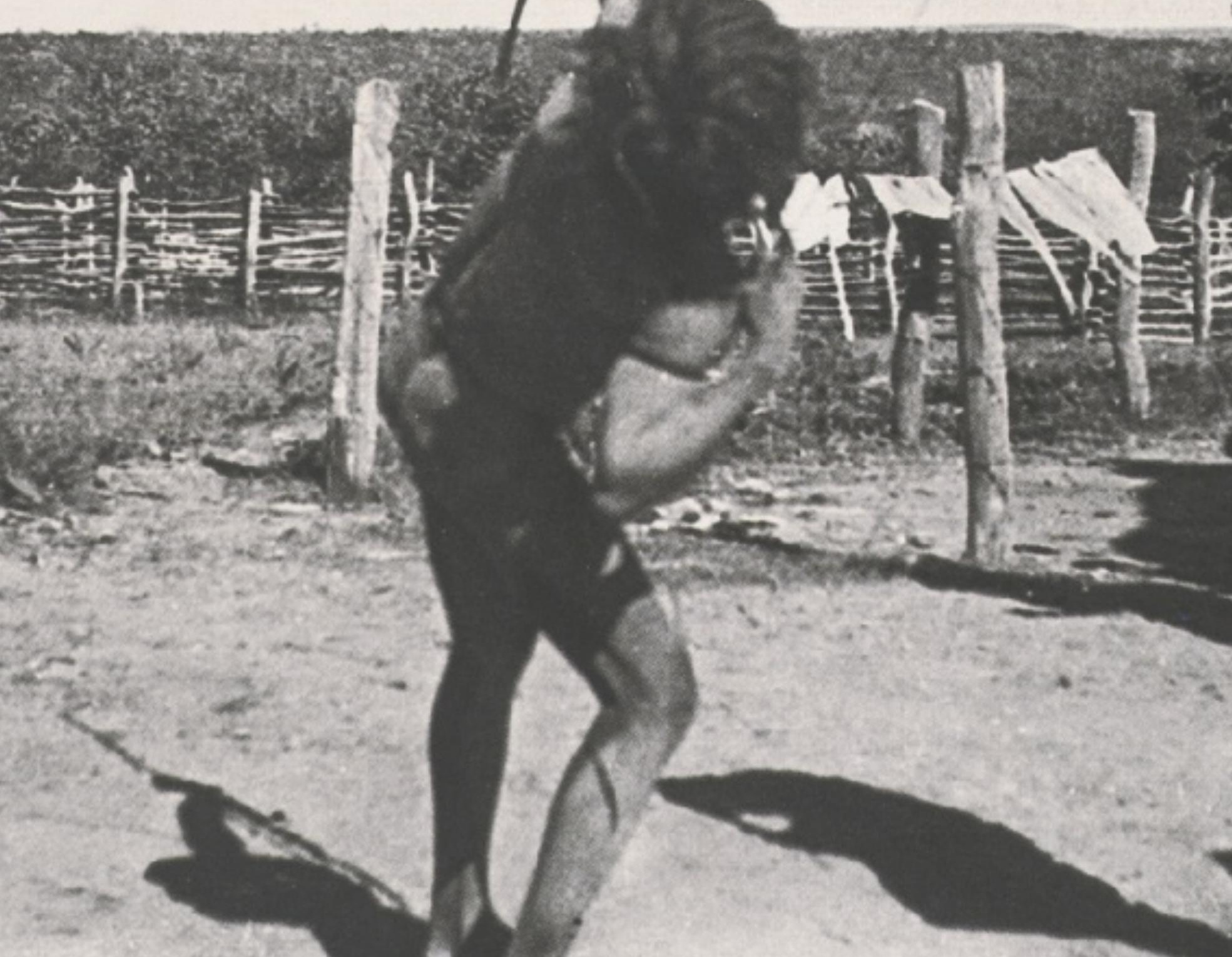

